\title{
Acetylation of TBX5 by KAT2B and KAT2A regulates heart and limb development
}

Tushar K Ghosh ${ }^{1 *}$, José J Aparicio-Sánchez ${ }^{1 *}$, Sarah Buxton ${ }^{1}$, Ami Ketley ${ }^{1}$, Tasabeeh Mohamed $^{1}$, Catrin S Rutland ${ }^{2}$, Siobhan Loughna ${ }^{1}$ and J. David Brook ${ }^{1 * *}$

${ }^{1}$ School of Life Sciences, Queen's Medical Centre, University of Nottingham, Nottingham, NG7 2UH, UK;

${ }^{2}$ The School of Veterinary Medicine and Science, Sutton Bonington Campus, Sutton Bonington, University of Nottingham, LE12 5RD, UK

*These authors contributed equally

**To whom correspondence may be addressed: J David Brook, School of Life Sciences, University of Nottingham, Nottingham, NG7 2UH, UK; Tel: +44-115- 8230345; Email: David.Brook@nottingham.ac.uk

Key words: TBX5, acetylation, heart development, CRISPR-Cas, zebrafish 


\begin{abstract}
TBX5 plays a critical role in heart and forelimb development. Mutations in TBX5 cause Holt-Oram syndrome, an autosomal dominant condition that affects the formation of the heart and upper-limb. Several studies have provided significant insight into the role of TBX5 in cardiogenesis; however, how TBX5 activity is regulated by other factors is still unknown. Here we report that histone acetyltransferases KAT2A and KAT2B associate with TBX5 and acetylate it at Lys339. Acetylation potentiates its transcriptional activity and is required for nuclear retention. Morpholino-mediated knockdown of kat $2 a$ and kat $2 b$ transcripts in zebrafish severely perturb heart and limb development, mirroring the tbx5a knockdown phenotype. The phenotypes found in MO-injected embryos were also observed when we introduced mutations in the kat $2 a$ or kat $2 b$ genes using the CRISPR-Cas system. These studies highlight the importance of KAT2A and KAT2B modulation of TBX5 and their impact on heart and limb development.
\end{abstract}

\title{
INTRODUCTION
}

TBX5 is essential for heart and fore-limb development and mutations in this gene result in defective structures (Li et al. 1997, Basson et al. 1997). During heart development a number of cardiac transcription factors, most notably GATA4 and MEF2C, physically associate and functionally cooperate with TBX5 in order to control and regulate many processes taking place during cardiogenesis (Garg et al. 2003, Ghosh et al. 2009). In addition to this, recent studies suggest that overexpression of these three factors is sufficient to reprogram fibroblast cells to cardiomyocyte-like cells (Ieda et al. 2010). Even though the role of TBX5 in cardiac development and reprogramming has been extensively studied, how TBX5 activity is regulated by other factors remains largely unknown.

Acetylation of histone and non-histone proteins regulates cellular physiology and affects cellular proliferation, growth and differentiation (Parrie et al. 2013). The acetylation and deacetylation status of histones ultimately controls muscle differentiation and cardiac hypertrophy (McKinsey et al. 2001, Backs and Olson 2006). Their activity on defined nonhistone proteins has also been described (Thisse and Thisse 2008, Chernyavskaya et al. 2012). Two major histone acetyltransferases (HATs) in mammals are KAT2B (PCAF) and p300. Although functionally similar they have distinct roles in muscle differentiation (Puri et al. 1997). p300 is essential for embryogenesis and mice lacking a functional p300 gene die between 9 and 11.5 days of gestation. These knockout mice exhibit defects of cardiac muscle differentiation and trabeculation (Yao et al. 1998). Furthermore, transgenic expression of p300 in the adult heart results in hypertrophy and heart failure (Yanazume et al. 2003). Kat2a, a paralogue of Kat2b shares similar sequences and enzymatic activity. Although they are 73\% identical and possess similar acetyltransferase activity, they have distinct but overlapping roles in mouse embryogenesis. Kat2a-null embryos die during embryogenesis, whilst Kat $2 b$ knockout mice show no obvious phenotype. Combined loss of Kat $2 a$ and Kat $2 b$ in mice leads to even more severe developmental defects, suggesting a partial functional redundancy between Kat $2 a$ and Kat2b in vivo (Xu et al. 2000). The obvious functional disparity between 
$K a t 2 b$ and Kat $2 a$ may be partly due to their differential expression pattern during early embryogenesis, as Kat $2 b$ is expressed slightly later than Kat2a (Yamauchi et al. 2000). $K a t 2 b$ and Kat $2 a$ are ubiquitously expressed and most abundant in skeletal muscle. The former is also highly abundant in the heart, whereas the latter is highly abundant in pancreas (Yang et al. 1996).

Acetylation of cardiac transcription factors GATA4 and MEF2C is important for the differentiation of cardiomyocytes (Miska et al. 2001, Kuninger et al. 2007). Indeed, acetylation of GATA4 triggers the differentiation of embryonic stem cells into cardiac myocytes (Borghi et al. 2001). Recent studies suggest p300 and Hdac3 regulate early cardiogenesis by modulating Tbx5 activity (Lewandowski et al. 2014). Here we report that both KAT2A and KAT2B physically associate with and acetylate TBX5 at Lys339. Acetylation augments its transcriptional activity and is required for its nuclear retention. Morpholino-mediated knockdown and CRISPR-Cas-mediated knockout of kat $2 a$ and kat $2 b$ in zebrafish produces both heart and fin phenotypes similar to that observed in $t b x 5 a$ morphants. This study reveals a role for KAT2A and KAT2B and their link with TBX5, in heart and limb development.

\section{RESULTS}

\section{TBX5 is acetylated by both KAT2A and KAT2B}

In view of the role of $\mathrm{p} 300$ in acetylation and transcriptional regulation of GATA4, MEF2C and TBX5 we investigated whether TBX5 could be acetylated and whether this has functional consequences. Cos7 cells were transiently transfected with plasmids encoding TBX5-FLAG along with KAT2B-HA or p300-FLAG. The FLAG-tagged TBX5 proteins were affinity purified from the cell lysates using anti-FLAG-agarose beads. The bound proteins were eluted using sample buffer, fractionated on SDS-PAGE gels and subsequently immunoblotted with anti-acetylated lysine antibody. As shown in Fig. 1A, TBX5 was specifically acetylated by KAT2B, whereas p300 failed to do so. Treatment with histone deacetylase inhibitor Trichostatin A (TSA) enhanced TBX5 acetylation. In order to further confirm the TBX5 acetylation by KAT2B, we also performed in vitro acetylation assays in cell-free extracts using purified MBP-TBX5 and KAT2B proteins. The results suggest that MBP- TBX5 is acetylated by KAT2B in vitro (Fig. S1).

To demonstrate the association between TBX5 and KAT2B, pull-down experiments were conducted on the cell extracts using anti-FLAG-agarose beads. Fig. 1B shows FLAG-tagged TBX5 specifically pulled down HA-KAT2B from the lysate in which both proteins were expressed suggesting their physical association. In addition we examined whether KAT2A, a functional homologue of KAT2B could also acetylate TBX5. Similar co-expression of KAT2A and TBX5 in Cos7 cells resulted in acetylation of TBX5 (Fig. 1C) and pull-down assays also identified their physical interaction (Fig. 1D).

In promoter-reporter assays, cotransfection of plasmids encoding TBX5, KAT2B and the reporter plasmid pGL3-MYH6-I resulted in significant enhancement of TBX5-mediated 
transcription. Likewise, KAT2A significantly enhanced the TBX5-mediated transcription from the MYH6 promoter (Fig. 1E). We also performed similar reporter assays on two other TBX5 target promoters: ANF and CX40. The results suggest that the ANF promoter activity is significantly enhanced in presence of KAT2B and TBX5 (Fig. 1F), whereas Cx40 promoter (-1190/+121-Luc) was not TBX5 responsive in this cell line (data not shown).

Overall, these experiments suggest that the acetylation of TBX5 is a conserved function shared between the KAT2-family of proteins.

\section{Mapping the acetylation residue in TBX5}

To map the acetylation residue in TBX5, the web-based prediction sites PredMod, PAIL and PHOSIDA were used. Whilst the predictions vary considerably in each case, Lys234 was picked up by all three sites (Fig. S2, A-C). However, conservative substitution of lysine 234 to arginine did not eliminate acetylation suggesting that Lys234 is not the key lysine residue for acetylation (Fig. S2D).

Next, we attempted to derive an acetylation motif for KAT2B and KAT2A. We searched in the literature for proteins that are acetylated by KAT2B and KAT2A, extracted short stretches of amino acid sequence either side of the key lysine residues and aligned them. Since previous studies suggested an acetylation motif of 3-4 amino acids we set a sliding window of six amino acids to the N-terminus and C-terminus of the relevant lysine residue. Frequency plot analysis of 25 acetylated peptide sequences suggested an enrichment of a lysine residue at position -3 , a lysine or arginine at position +1 and a serine, proline or lysine at +2 (Fig. 2A and Fig. S3A). Overall, the alignment provided a lysine rich consensus motif $\mathrm{K}-\mathrm{X}-\mathrm{X}-\mathrm{K}-\mathrm{K} / \mathrm{R}-\mathrm{P} / \mathrm{L} / \mathrm{K}$ (where $\mathrm{X}$ denotes any amino acids). For the control, we randomly picked similar lengths of peptide sequences around a lysine residues from 25 non-acetylated proteins in the UniProt database and no such enrichment was found (Fig. S3B).

On close scrutiny, we observed that only one third of the 25 targets contain the full consensus and many contain a partial motif either $\mathrm{K}-\mathrm{X}-\mathrm{X}-\mathrm{K}$ or $\mathrm{K}-\mathrm{K} / \mathrm{R}-\mathrm{P} / \mathrm{L} / \mathrm{K}$. These two partial motifs also overlap with the recognition sequences obtained for p300 and GCN5 (Gu and Roeder 1997, Rojas et al. 1999). Thus, we noted a significant enrichment of basic amino acid residues lysine or arginine and aliphatic amino acids proline or leucine around the key acetylated lysine.

Next, we examined TBX5 but were unable to find any obvious full-length acetylation motif. However, TBX5 does contain two partial motifs; K-R-K and K-K-P at positions 325-327 and 339-341, respectively (Fig. 2B). In order to ascertain whether any of these lysine residues could be acetylated, we made conservative substitutions of these lysine residues to arginine. We generated mutants K325R or K327R in the KRK motif, and K339R or K340R for the KKP motif and analysed whether they could be acetylated by KAT2B in Cos7 cells. Mutants K325R and K327R in KRK were efficiently acetylated by KAT2B (Fig. 2C). The mutant K340R was also acetylated by KAT2B. However, mutation of K339R completely eliminated acetylation indicating that the lysine 339 is the key residue for acetylation of TBX5 (Fig. 2D), 
A

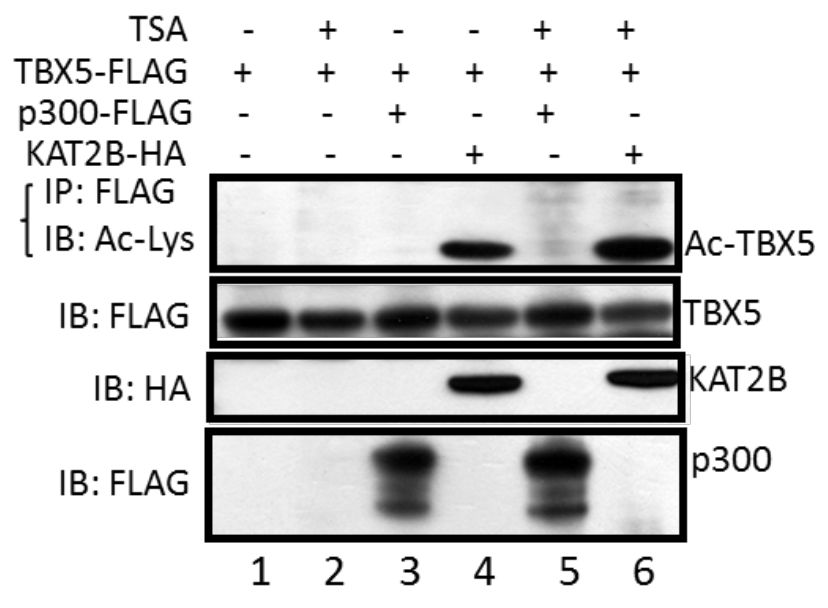

C

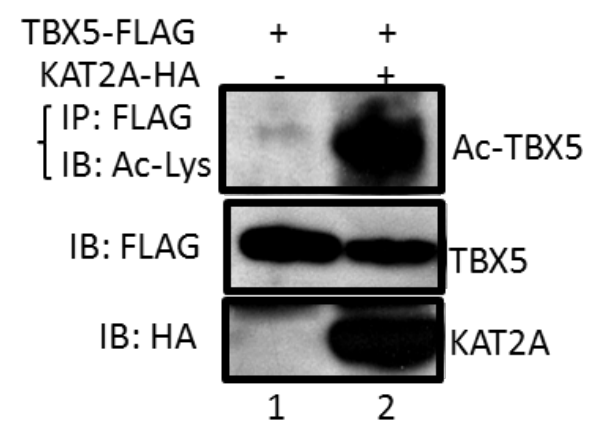

\section{$E$}

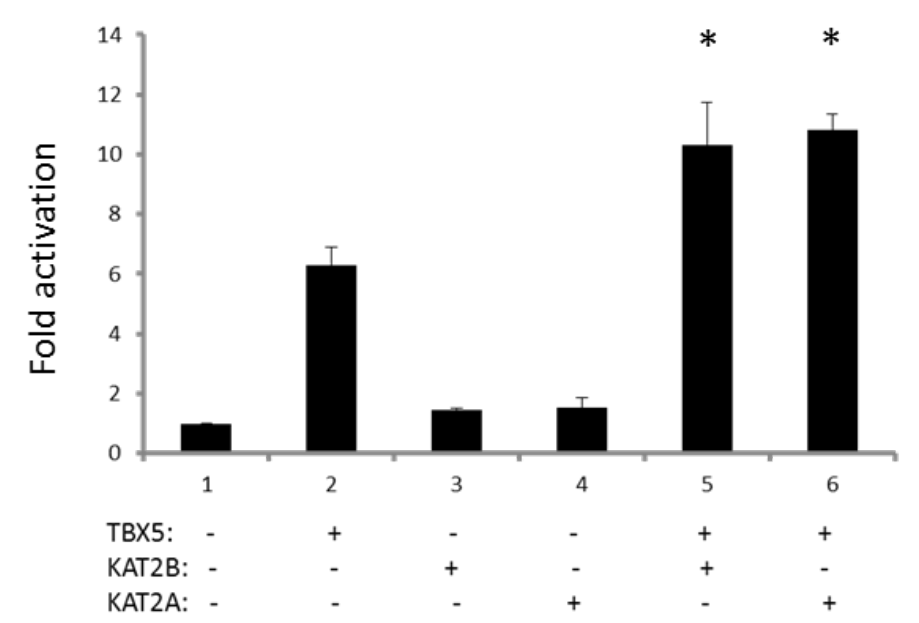

B

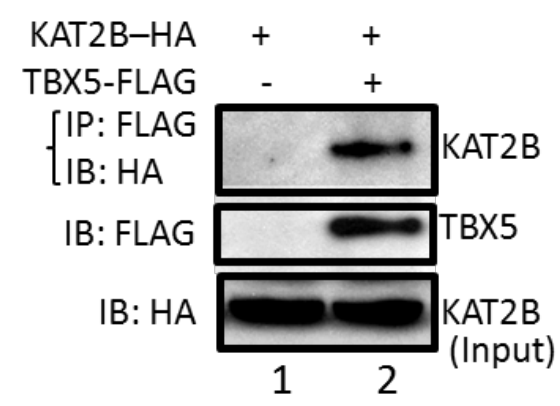

D

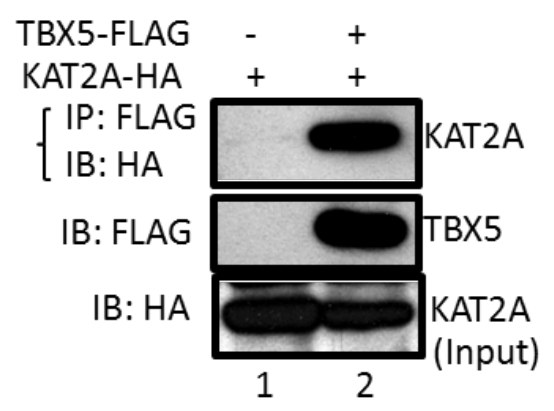

F

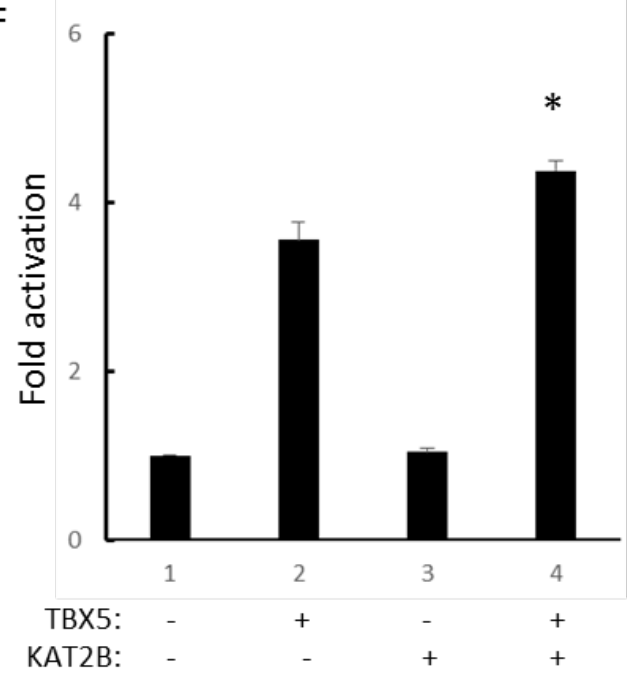

Figure 1. TBX5 associates with, and is acetylated by KAT2B and KAT2A. (A-D) Western blots of pull down assays show that (A) KAT2B acetylates TBX5 (lane 4 and 6), whereas p300 does not (lanes 3 and 5). TSA enhances the acetylation level of TBX5 (lane 6). (B) TBX5 and KAT2B physically associate. (C) TBX5 is acetylated by KAT2A. (D) Association of TBX5 and KAT2A. (E) and (F) Reporter assays show that KAT2B and KAT2A enhance the TBX5-mediated transcription on a MYH6 promoter (E) and on an ANF promoter (F). Results are from three independent experiments. Error bars represent $\mathrm{SD}, * \mathrm{P}<0.05$ (Student's t test). IP- immunoprecipitation, IB-immunoblot and TSA-Tricostatin A. 
an effect that was also seen in H9C2 cells (Fig. S3C). KAT2A, which also acetylates TBX5, failed to acetylate the mutant K339R as well (Fig. 2F). To determine whether TBX5K339R still retains its ability to interact with KAT2B, we performed pull-down assays and the result suggests that the mutant TBX5 K339R physically interacts with KAT2B (Fig. S3D).

Interestingly, K339 is well conserved among TBX5 proteins (Fig. 2B) which probably suggests its evolutionary significance. KAT2B also failed to potentiate the transcriptional activity of the K339R mutant in reporter assays (Fig. 2E), suggesting that acetylation indeed enhanced the transcriptional potential of TBX5. We also checked the native transcriptional activity of TBX5K325R, TBX5K327R, TBX5K339R and TBX5K340R on the MYH6 promoter. K325R, K327R and K340R mutants didn't show any significant change in their transcriptional activity in comparison with TBX5 WT. However, a significant reduction was observed in the K339R mutant (Fig. S3E).

Overall, these experiments suggest that TBX5 is acetylated at lysine 339 , and that this residue is critical to potentiate its transcriptional activity.

\section{Acetylation of TBX5 and nucleocytoplasmic shuttling}

TBX5 is a shuttling protein. It has two nuclear localization signal domains; NLS1 (aa78-90) and NLS2 (aa325-340) that act in a cooperative fashion to mediate nuclear translocation (Collavoli et al. 2003). Also, it has a nuclear export signal (NES, aa152-160) which is recognized by the CRM1 export protein (Kulisz and Simon 2008) (Fig. 3A). Since the acetylation residue K339 is located within the NLS2 we investigated whether acetylation would have an impact on TBX5 nuclear localization on two different cell lines: Cos7 and C2C12 cells. In Cos7 cells, wild type TBX5 was expressed exclusively in the nucleus in 95\% of cells counted. In the case of the acetyl-deficient mutant TBX5-K339R, only $24 \%$ of the cells showed a TBX5-K339R nuclear localization, whereas 76\% showed a cytoplasmic localization (both nuclear and cytoplasmic or predominantly cytoplasmic). In most cells we also found accumulation of TBX5-K339R around the outer nuclear membrane. We found similar results when we transfected TBX5-WT or TBX5-K399R in C2C12 cells: TBX5-WT showed an exclusive nuclear localization in $94 \%$ of $\mathrm{C} 2 \mathrm{C} 12$ cells, whereas only a $17 \%$ of cells showed a nuclear TBX5-K339R localization $(83 \%$ of the cells presented a cytoplasmic localization) (Fig. 3B and S4A).

In order to confirm that this impairment in cellular localization is indeed due to the lack of acetylation, we treated TBX5 WT-transfected Cos7 cells with Garcinol, a potent inhibitor of HATs KAT2B and p300. When cells were treated with $20 \mu \mathrm{M}$ of Garcinol, only $50 \%$ of them showed a nuclear TBX5-WT localization, whereas 50\% showed a cytoplasmic distribution (Fig. S4B).

These results suggested that the lack of acetylation in the mutant TBX5-K339R leads to an impaired cellular localization. In order to determine whether TBX5 acetylation is required for its nuclear import or for nuclear retention, we treated cells transfected with either TBX5-WT 
A

Acetylated proteins

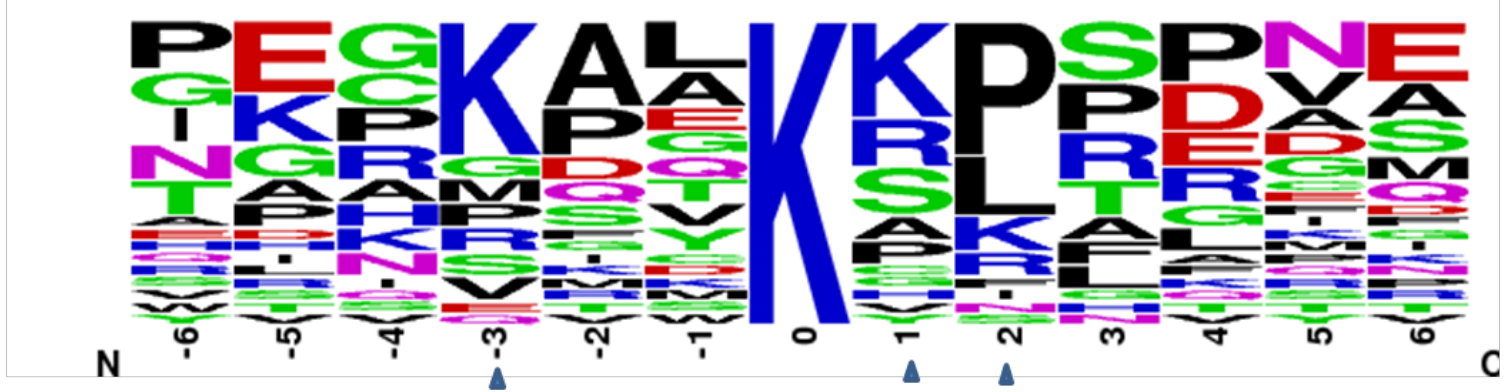

Consensus: $\mathrm{KXXKK} \underline{\mathrm{K}} / \mathrm{RP} / \mathrm{L} / \mathrm{K}$

B

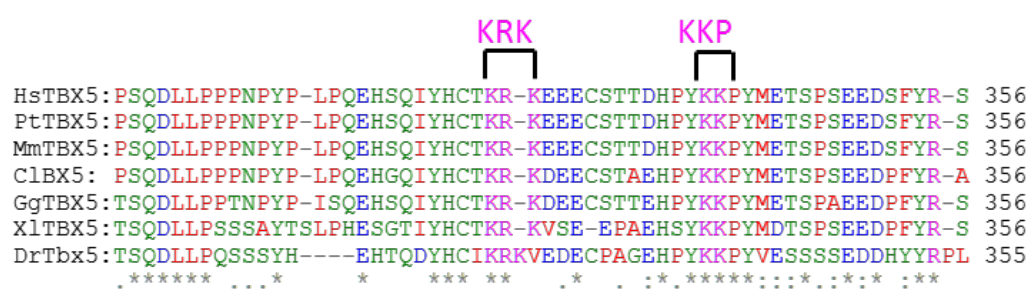

C

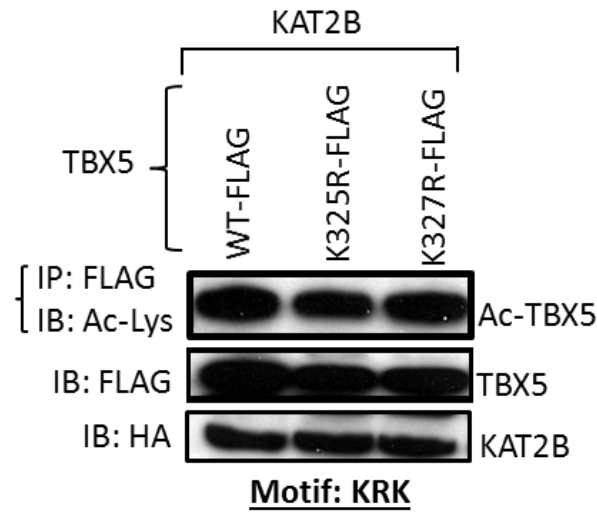

$E$

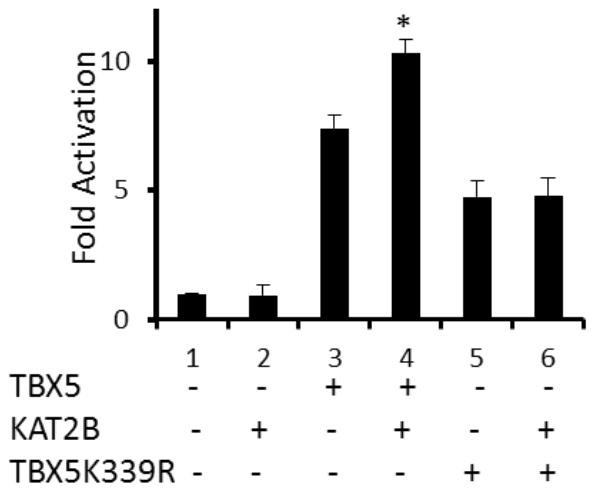

D

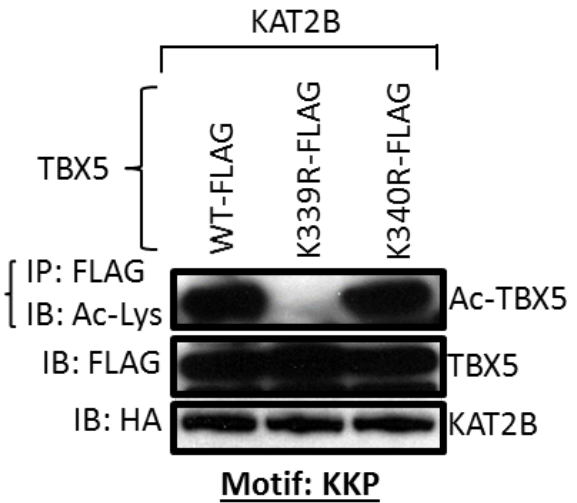

$\mathrm{F}$

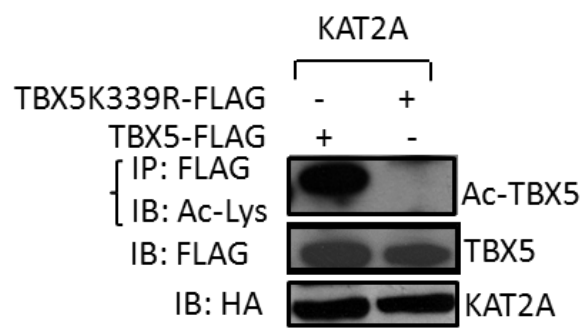

Figure 2. Mapping the acetylation residue in TBX5. (A) The sequence logo plot shows the frequencies of amino acids at positions -6 to +6 with reference to the acetylated lysine residue. Arrows show the positions of enrichment. (B) Alignment of TBX5 protein sequences showing conservation of the KRK and KKP motifs. Hs-Human, Pt-chimpanzee, Mm-mouse, ClDog, Gg-chicken, Xl-xenopus and Dr-zebrafish. (C and D) Pull-down assays to map the key lysine residue for acetylation in KRK (C) and KKP (D) motifs. (E) Reporter assay shows KAT2B failed to potentiate the transcriptional activity of the acetyl-deficient mutant protein TBX5 K339R on a MYH6 promoter. Results are from three individual experiments. Error bars represent $\mathrm{SD},{ }^{*} \mathrm{P}<0.05$. (F) Pull-down assay showing KAT2A does not acetylate TBX5K339R. 
or TBX5-K339R with leptomycin B, a potent inhibitor of the exportin CRM1, which mediates TBX5 nuclear export (Kulisz and Simon 2008). Following leptomycin B treatment, we found that TBX5-K339R remained inside the nucleus in $96 \%$ of Cos7 cells analysed and in $85 \%$ of $\mathrm{C} 2 \mathrm{C} 12$ cells, both in contrast to the predominantly cytoplasmic localization of TBX5-K339R in non-treated cells. Treatment of TBX5-WT with leptomycin B did not have any effect on its normal nuclear localization (Fig. 3C and S4A). We also checked the potential co-localization of TBX5-K339R and endogenous CRM1 outside the nucleus. Immunofluorescence staining of TBX5-K339R and endogenous CRM1 showed that they both co-localize outside the nucleus, mainly around the outer nuclear membrane. When TBX5-WT was transfected, or when TBX5-K339R was treated with Leptomycin B, both TBX5 and CRM1 were localized strictly inside the nucleus (Fig. S4C).

Finally, we also performed in vitro interaction studies on CRM1 and TBX5-WT or TBX5K339R. Following transfection of TBX5-WT-FLAG or its mutant variant TBX5-K339RFLAG in Cos7 cells (in which CRM1 is ubiquitously expressed), a pull-down assay revealed that the acetyl-deficient variant TBX5-K339R presents higher affinity for the CRM1 protein than its WT counterpart (Fig. 3D and 3E).

Overall, these results indicate that acetylation of TBX5 is required for its nuclear retention and to maintain a critical concentration of TBX5 inside the nucleus, whereas lack of acetylation promotes its nuclear export via the CRM1-pathway.

\section{Knockdown of $k a t 2 a$ and $k a t 2 b$ in zebrafish produce heart defects}

Since both KAT2A and KAT2B interact with and acetylate TBX5, we looked for their in vivo functional impact on heart and limb development in zebrafish. Zebrafish KAT proteins share a high degree of similarity with their human and murine counterparts $(84 \%$ and $85 \%$ for KAT2A, and $81 \%$ and $80 \%$ for KAT2B respectively) as revealed by amino acid sequence analysis using the ClustalW2 software (Fig. S5A). In addition to this, we checked the expression pattern of kat $2 a$ and $k a t 2 b$ in zebrafish, for which we dissected out head/thorax containing heart and tail regions at different stages of development. RNAs were extracted and RT-PCR analysis revealed expression of both kat $2 a$ and kat $2 b$ in the heart and tail regions throughout developmental stages 36-72hpf (Fig. S5B). ISH analysis using kat2a and $k a t 2 b$ probes on $48 \mathrm{hpf}$ embryos indicated that both of them are mainly expressed in the head and cardiac region in an ubiquitous manner, as expected. We also detected expression of both transcripts in the tail region and in the pectoral fins (Fig. S5C).

To analyse the in vivo roles of kat2a and kat $2 b$ we carried out morpholino-mediated knockdown studies in zebrafish with both translation blocking and splice blocking morpholinos (mismatch morpholinos were employed as controls). The embryos were screened for heart phenotypes and based on the severity of heart defects they were assigned to one of three categories: normal, mild or severe. In normal embryos, both the atrium and the ventricle were clearly defined and tightly packed into a figure eight configuration. In the mild phenotype the atrium and ventricle were clearly distinguishable, but looping was noticeably 
relaxed and slight pericardial oedema was usually seen, as well as a slight reduction in the rate of heart beat.

A

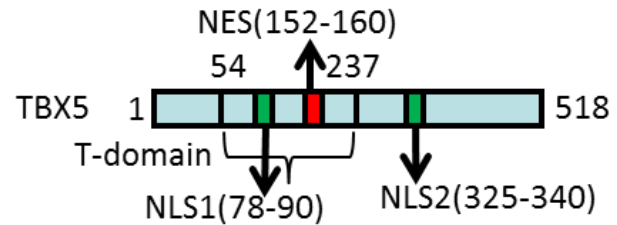

B
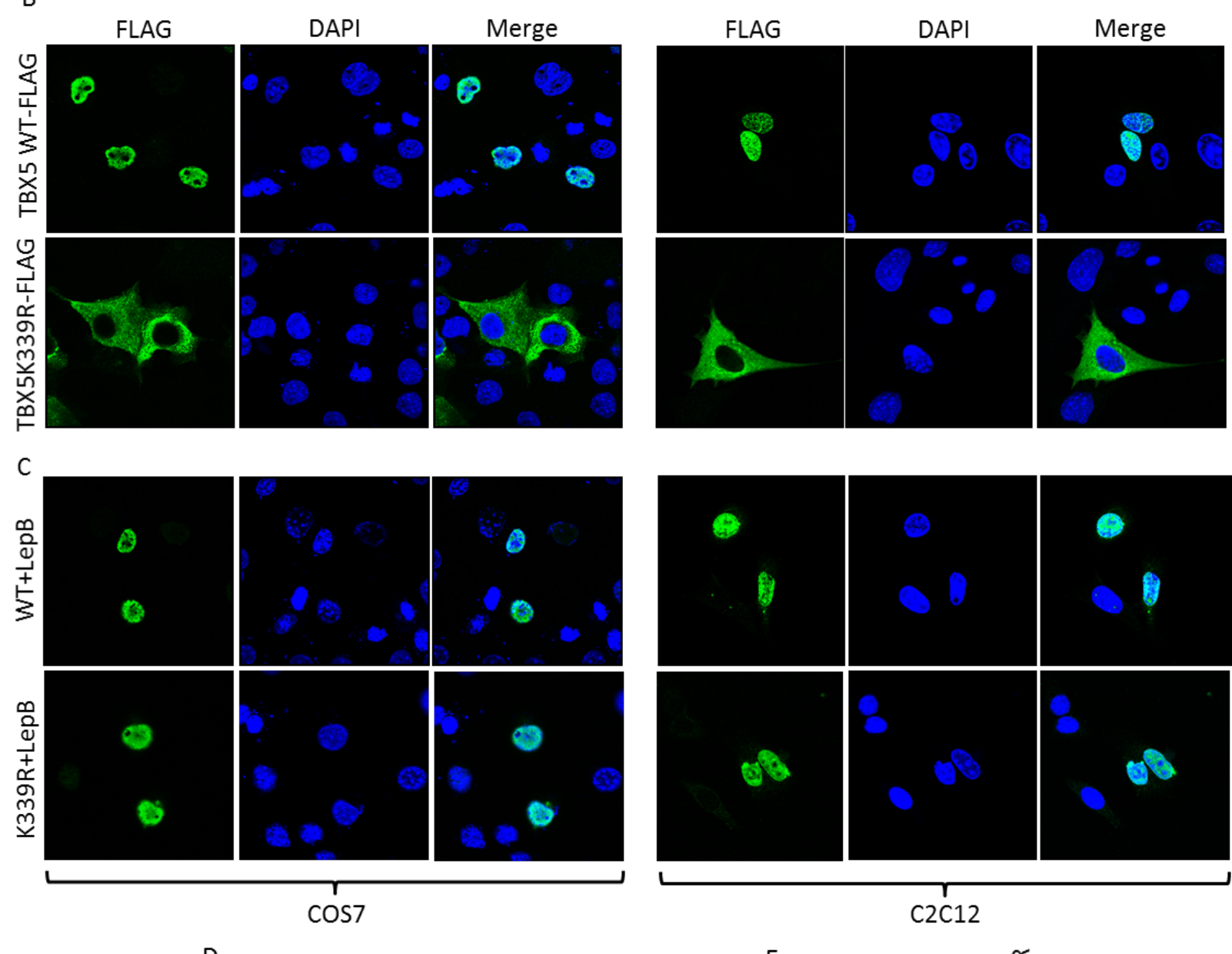

$\mathrm{E}$
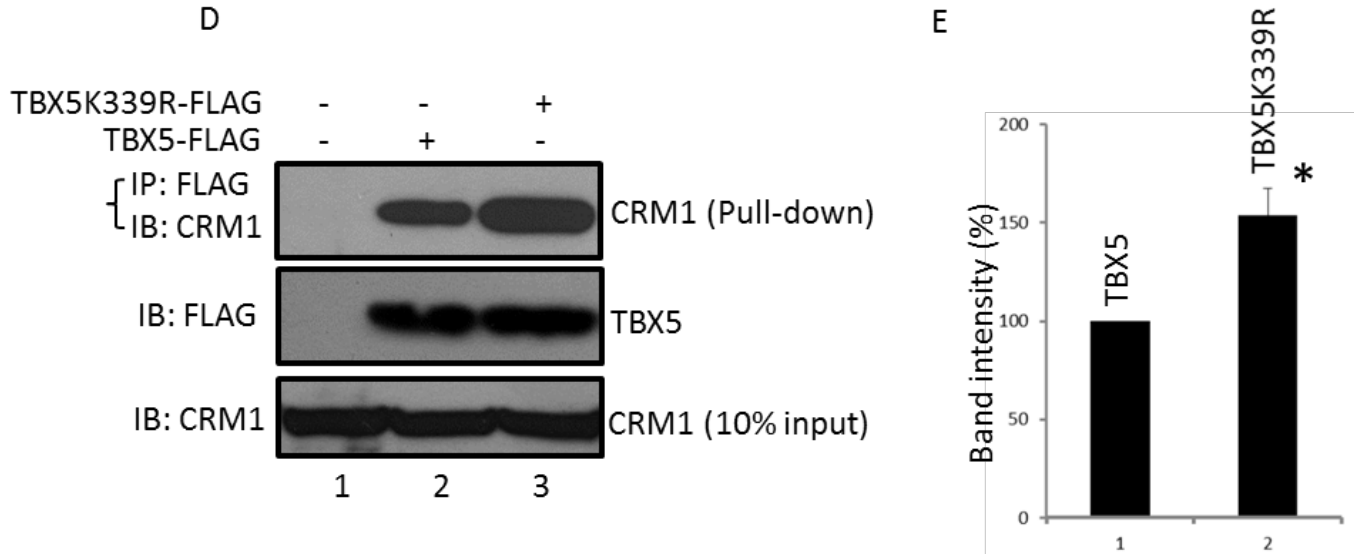

Figure 3. Acetylation and nucleocytoplasmic shuttling of TBX5. (A) Schematic representation of TBX5. NLS1-Nuclear Localization Signal domain 1, NLS2-Nuclear Localization Signal domain 2 and NES- Nuclear Export Signal. (B) Representative images of the cellular distribution of TBX5-WT and TBX5-K339R in both COS7 and C2C12 cells. (C) The 
effect of leptomycin B on nuclear/cytoplasmic distribution of TBX5-WT and TBX5-K339R. (D) Pull-down assay showing increased affinity of mutant protein TBX5K339R for CRM1. (E) Quantification of CRM1 protein pull-down by wildtype (TBX5-FLAG) and acetyl-deficient mutant protein (TBX5K339R-FLAG). Results are mean \pm SD from 3 individual experiments, ${ }^{*} \mathrm{P}<0.001$, Student's t test.

In the severe phenotype the atrium and ventricle were hardly distinguishable and they were irregular in shape and/or size, and the heart in general was stretched to a thin tube. Looping was lost, a moderate-large pericardial oedema is observed and the heart rate was notably slower.

Overall, kat $2 a$ and kat $2 b$ morpholinos produced very similar cardiac developmental defects to those observed in $t b x 5 a$ morphants (Fig. 4A). In both kat2a and kat $2 b$ knockdown embryos heart looping was interrupted and pericardial oedema was seen, as was altered chamber morphology. In the case of kat $2 a$ and kat $2 b$ single knockdowns, we found that $44 \%$ and $45 \%$ of injected embryos presented a mild heart phenotype respectively, whereas only a $12 \%$ and $20 \%$ showed a severe phenotype. However, in tbx5a knockdown $57 \%$ of the injected embryos showed a severe heart phenotype, with only $19 \%$ of them presenting a mild phenotype. A similar proportion of mild and severe phenotypes was found when we injected both kat $2 a$ and kat $2 b$ morpholinos together (29\% and $48 \%$ respectively), thus supporting a potential compensatory effect between kat $2 a$ and kat2b (Fig. 4B). Defects were also seen affecting other areas (e.g fins, somite, brain, etc) in kat $2 a$ and kat $2 b$ knockdowns.

To demonstrate kat $2 a$ and kat $2 b$ knockdown, RT-PCR was performed on the RNA samples obtained from the splice knockdown morphants. Agarose gel electrophoresis confirmed that splicing of exon 2 was affected in kat $2 a$ and kat $2 b$ transcripts of the morphants. In both cases a normal size allele ( $345 \mathrm{bp}$ for kat $2 \mathrm{a}$ and $342 \mathrm{bp}$ for $k a t 2 b$ respectively) and a short isoform lacking exon 2 (221bp for kat $2 a$ and $204 \mathrm{bp}$ for kat $2 b$ respectively) were detected (Fig. 4C-D). The spliced products were also sequence verified. Overall, these experiments suggest that $k a t 2 a / 2 b$ knockdown produced heart phenotype similar to those in tbx5a morphants, suggesting a genetic link between them and the presence of a compensatory effect between $k a t 2 a$ and $k a t 2 b$.

\section{Knockout of $k a t 2 a$ and $k a t 2 b$ in zebrafish phenocopies the heart defects found in kat $2 a$ and $k a t 2 b$ knockdowns}

To validate the kat $2 a$, kat $2 b$ and $t b x 5 a$ loss-of-function phenotypes, we generated F0 mutants for each of these genes using the CRISPR-Cas genome-editing technology. sgRNAs targeting $k a t 2 a$, kat $2 b$ and $t b x 5 a$ exonic regions were designed using the CHOPCHOP web tool (Fig. S6A) and injected alongside Cas9 protein into 1-cell stage zebrafish embryos (Cas9-only injected embryos were used as negative controls). Screening $48 \mathrm{hpf}$ revealed that $43 \%$ of embryos injected with Cas9 and kat $2 a$ sgRNA, and 39\% of embryos injected with Cas 9 and $k a t 2 b$ sgRNA, presented a mild heart phenotype very similar to that found in their knockdown counterparts; whereas $6 \%$ and $5 \%$ presented a severe phenotype respectively. Injection of Cas 9 and a $t b x 5 a$ sgRNA led to $17 \%$ of the embryos showing a mild phenotype, and $43 \%$ with a severe phenotype (Fig. $5 \mathrm{~A}$ and $5 \mathrm{~B}$ ). In order to confirm the presence of mutations in embryos showing a cardiac phenotype, we performed a T7 assay and Sanger 
sequencing. These two approaches confirmed the presence of indels in $95-100 \%$ of analysed embryos for each gene $(\mathrm{n}=40)$, whereas injected embryos presenting a normal cardiac phenotype did not harbour any mutation in $90-95 \%$ of the cases $(n=20$ for each gene) (Fig. S6B and S6C). Analysis of

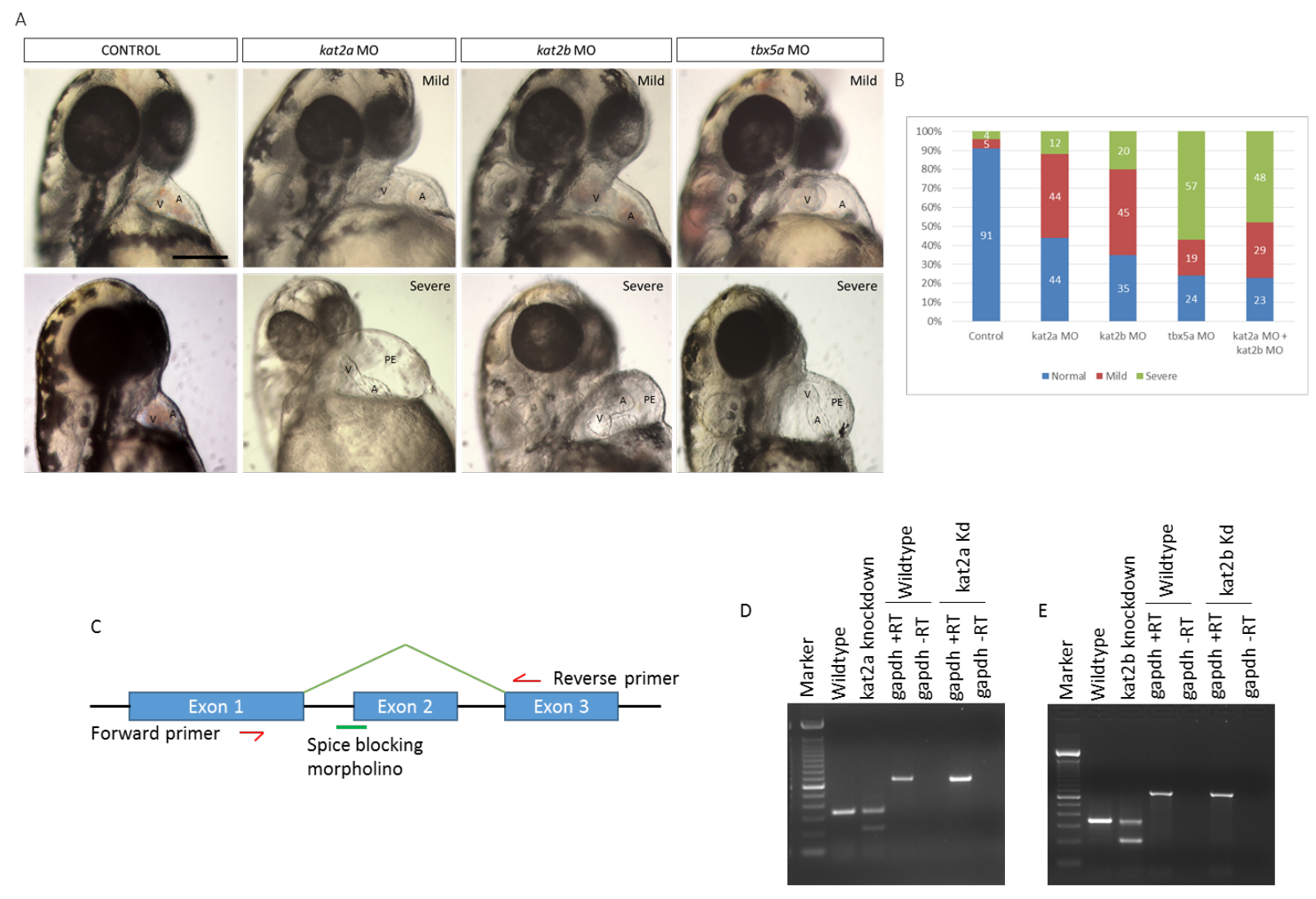

Figure 4. Morpholino-mediated knockdown of kat2a and kat $2 b$ in zebrafish present a heart phenotype similar to tbx $5 \mathrm{a}$ knockdown. (A) Zebrafish embryos showing the effect of tbx5, kat2a and kat2b morpholinos on heart development. Embryos were categorized as mild or severe based on the severity of the cardiac phenotype (V=ventricle, $A=$ atrium, $\mathrm{PE}=$ pericardial oedema). (B) Histogram showing the distribution of cardiac phenotypes based on severity in injected embryos ( $\mathrm{n}=200$ embryos per injection batch). (C) Design of the splice junction morpholinos at intron 1 and exon 2 boundaries of kat $2 b$ and $-2 a$. (D and E) Agarose gels showing the specific effect of splice blocking morpholinos against kat2a (D) and kat2b (E). Scale bar $=200 \mu \mathrm{m}$.

individual alleles indicated that in embryos presenting a cardiac phenotype, the rate of mutagenesis was around $80-90 \%$ with each of the sgRNAs, suggesting the presence of biallelic mutations in a substantial number of cells (Fig. 5B and Fig. S6C).

Next, we sought further insight into the cardiac phenotype present in kat2a and kat $2 b \mathrm{KO}$ in comparison to the $t b x 5 a \mathrm{KO}$ phenotype. In-situ hybridization using a $c m l c 2$ probe revealed that cardiac chamber morphology was slightly altered in mild embryos, and severely affected in severe embryos. Cardiac looping angle measurement indicated slight relaxation in kat $2 a$, $k a t 2 b$ and $t b x 5 a \mathrm{KO}$ embryos to a similar extent $\left(29^{\circ}, 26^{\circ}\right.$ and $36^{\circ}$ respectively) when compared to wildtype embryos $\left(16^{\circ}\right)$. This relaxation in cardiac looping was aggravated in $k a t 2 a, k a t 2 b$ and $t b x 5 a$ embryos showing a severe phenotype, where the heart was merely a stretched tube $\left(56^{\circ}, 54^{\circ}\right.$ and $64^{\circ}$ respectively) (Fig. 5C). 
Heart rate measurement also revealed the presence of bradycardia in $\mathrm{KO}$ embryos. At $48 \mathrm{hpf}$, heart rate for kat $2 a, k a t 2 b$ and $t b x 5 a \mathrm{KO}$ mild embryos was slightly slower compared with controls (119, 111 and 109 vs 135 bpm respectively). In embryos showing a severe cardiac phenotype for all three knockouts, heart rate was severely diminished in comparison with control embryos (90, 93 and 87 vs 135 bpm) (Fig. 5D).

Taking all these results together, the cardiac phenotype found in kat2a, kat2b and tbx5a knockdown embryos was validated in embryos harbouring mutations generated by the CRISPR-Cas9 genome-editing system. Furthermore, analysis of the cardiac phenotype in KO embryos confirmed the similarity between kat $2 a / k a t 2 b$ and $t b x 5 a$ mutants in terms of cardiac morphology, looping degree and rate of heart beat.

A
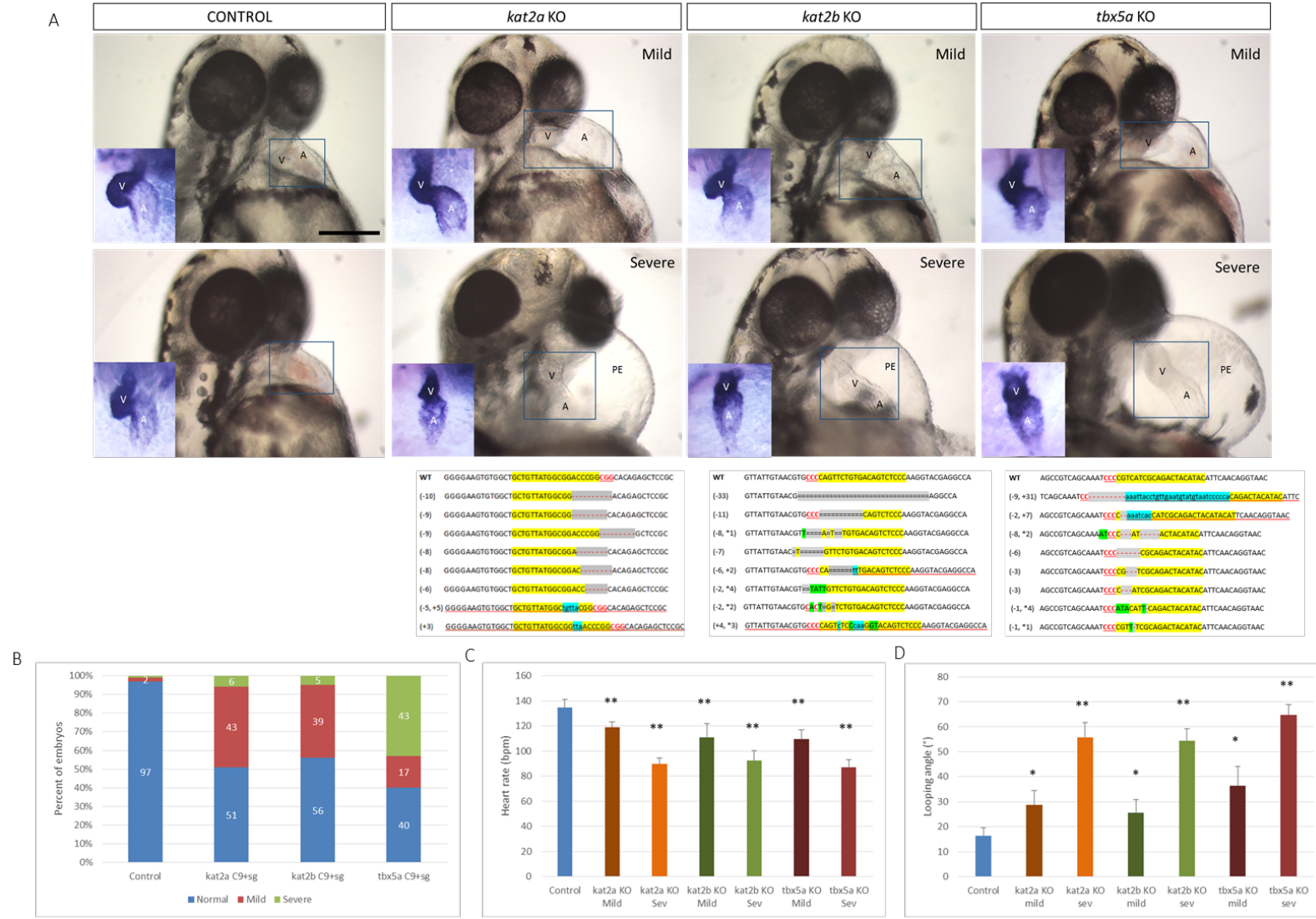

Figure 5. CRISPR-Cas-mediated knockout of kat2a, kat2b and tbx5a in zebrafish phenocopy their knockdown counterparts. (A) Images of $48 \mathrm{hpf}$ control embryos (injected with Cas9 only) and embryos injected with Cas9 and a sgRNA for kat2a, kat $2 \mathrm{~b}$ or tbx $5 \mathrm{a}$ respectively. Embryos were categorized as mild or severe based on the severity of the cardiac phenotype. The heart is highlighted in a blue square, and representative in-situ hybridization images using a cmlc2 probe are shown for each phenotype (bottom left corner, $\mathrm{V}=$ ventricle, $\mathrm{A}=$ atrium, $\mathrm{PE}=$ pericardial oedema). A list of some of the mutations found in kat2a, kat2b and tbx5a injected embryos is shown under the relevant embryos. Target site is highlighted in yellow, and PAM sequence is red underlined. Deletions are highlighted in grey, insertions in blue and substitutions in green. (B) Distribution of cardiac phenotypes based on severity in injected embryos ( $n=200$ embryos per injection batch). (C) Heart beating rate was measured in $48 \mathrm{hpf}$ embryos, indicating the presence of bradycardia in kat2a, kat2b and tbx5a KOs. (D) Average looping angle in $48 \mathrm{hpf}$ control and KO embryos. For (C) and (D), data + SEM is represented ( $\mathrm{n}=10-20$ embryos per phenotype). Relative to controls, $\mathrm{KO}$ embryos were statistically significant $(* * \mathrm{P}<0.001$, or $* \mathrm{P}<0.01)$. Scale bar $=200 \mu \mathrm{m}$

\section{Kat2a and $k a t 2 b$ KDs/KOs present abnormal fin development}


In addition to playing a critical role in heart development, $t b x 5 a$ is also a crucial regulator during fin development in zebrafish. Thus, both tbx5a knockout and knockdown embryos showed abnormal fin development $72 \mathrm{hpf}$ : in both cases, between a 40-60\% of the embryos lacked both pectoral fins, and approximately $15-20 \%$ of them had stumpy, shortened fins. In the case of $k a t 2 a$ and $k a t 2 b \mathrm{KOs} / \mathrm{KDs}$, around a $30-40 \%$ of the injected embryos presented the same kind of stumpy fins found in $t b x 5 a$ mutants, whereas only a 5-10\% showed no fins. In double knockdown embryos for kat $2 a$ and kat $2 b$, the percentage of embryos lacking both fins is increased up to $36 \%$, which is much closer to the proportion of $t b x 5 a$ mutants showing this phenotype (55\%) (Fig. 6A and 6B).

To further confirm the similarities between the tbx5a and kat2a/kat2b fin phenotype, we performed in-situ hybridization using a bmp4 probe, a typical fin marker which is also a tbx $5 a$ target gene during zebrafish development. In embryos showing stumpy fins for all 3 KOs bmp4 expression level was significantly lower than in control embryos and fin size was smaller; whereas embryos lacking fins showed no bmp4 expression at all (Fig. 6C).

Altogether, these results indicate that kat2a/kat $2 b \mathrm{KOs} / \mathrm{KDs}$ also present an abnormal fin phenotype comparable to that in the $t b x 5 a$ mutant, further supporting a potential genetic link in vivo between them.

\section{HAT inhibitors also produce cardiac and fin defects during zebrafish development}

To further substantiate the role of acetylation during zebrafish development, we used Garcinol to check whether the phenotypes produced by kat $2 a / k a t 2 b$ KOs are recapitulated by inhibiting the acetyltransferase activity. Following drug tritation, we found that treatment of zebrafish embryos with $100 \mu \mathrm{M}$ of Garcinol for 24 hours (starting at $20 \mathrm{hpf}$ ) lead to cardiac and fin defects similar to those found in morpholino KDs and CRISPR-Cas KOs. When treated with Garcinol, $84 \%$ of the embryos presented a mild cardiac phenotype respectively, whereas only a 3\% showed a severe cardiac phenotype (Fig. 7A and 7B). Further analysis also confirmed the presence of bradycardia and relaxation of cardiac looping in treated embryos (Fig 7C and 7D). With regards to fin development, embryo screening revealed that $75 \%$ of embryos treated with Garcinol showed stumpy, shortened fins similar to those found in KDs and KOs (Fig. 7A). Similar results were also found when we used a different HAT inhibitor such as anacardic acid (data not shown). Overall, these results suggest the chemical inhibition of $k a t 2 b$ also leads to cardiac and fin defects during zebrafish development, thus further substantiating the role of acetylation in this process.

\section{Tbx5a target genes are down-regulated in kat2a and kat2b KOs}

In order to check the misregulation of $t b x 5 a$ activity in vivo, we carried out qPCR analysis on $32 \mathrm{hpf} k a t 2 a$ and kat $2 b \mathrm{KO}$ embryos. We checked the relative mRNA levels of five different $t b x 5 a$ transcriptional targets: $f g f 10, b m p 4, n p p a$, hey 2 and $t b x 2 b$. As shown in Fig. 7A, all five genes were significantly down-regulated in both kat2a and kat $2 \mathrm{~b} \mathrm{KO}$ embryos. To further confirm the down-regulation of tbx5a target genes, we performed in-situ hybridization on 32 hpf kat2a and kat2b KO embryos using probes for $f g f 10$ (a fin marker), bmp 4 and nppa 
(two different cardiac markers). ISH on $f g f 10$ showed a down-regulation of this gene in kat2a/kat2b KOs in comparison to their WT counterparts in the pectoral fins among other tissues. In the case of $n p p a$ and bmp4, their expression levels were also reduced in the heart of KO embryos in comparison with their WT counterparts (Fig. 7B).
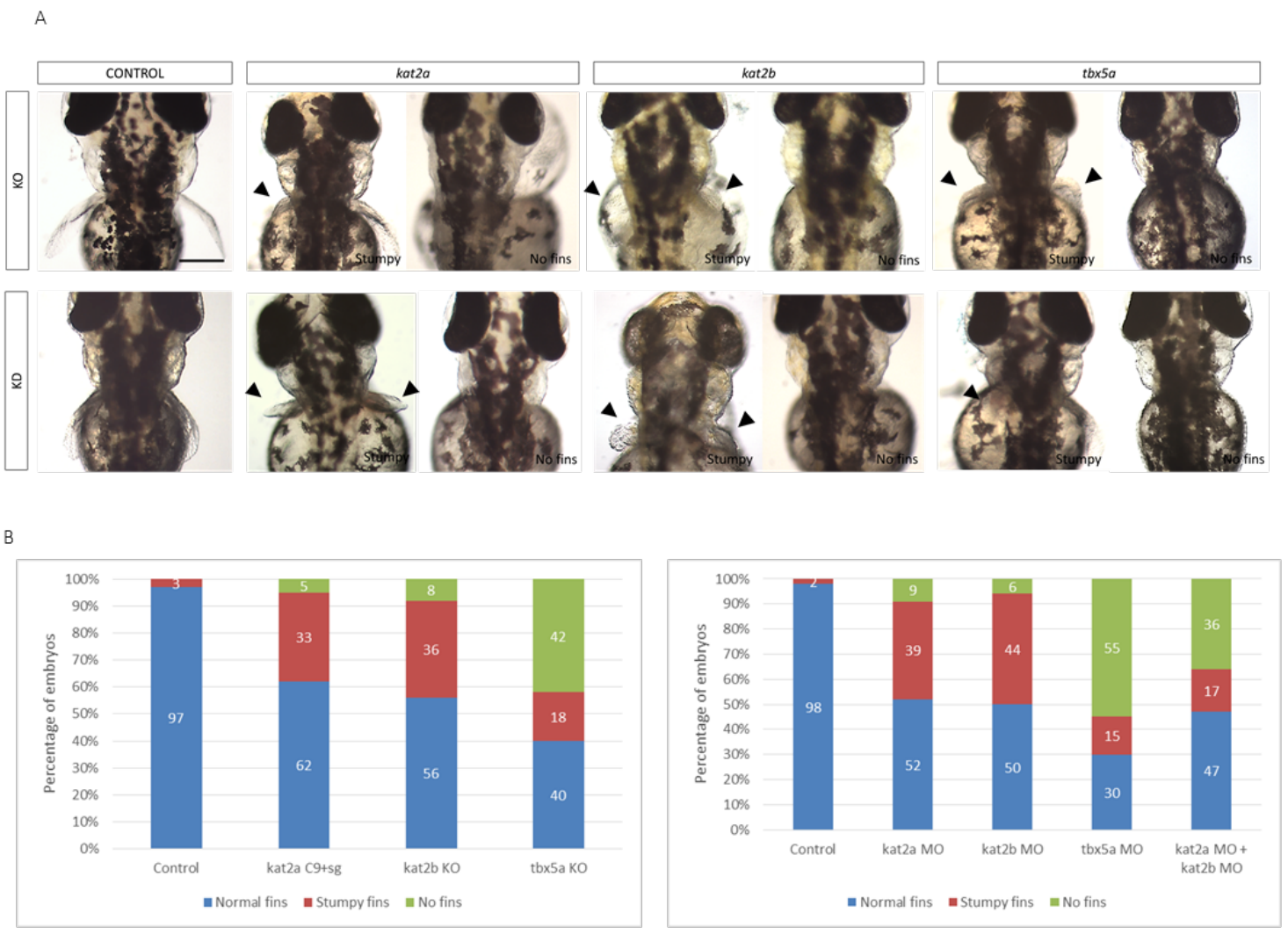

C
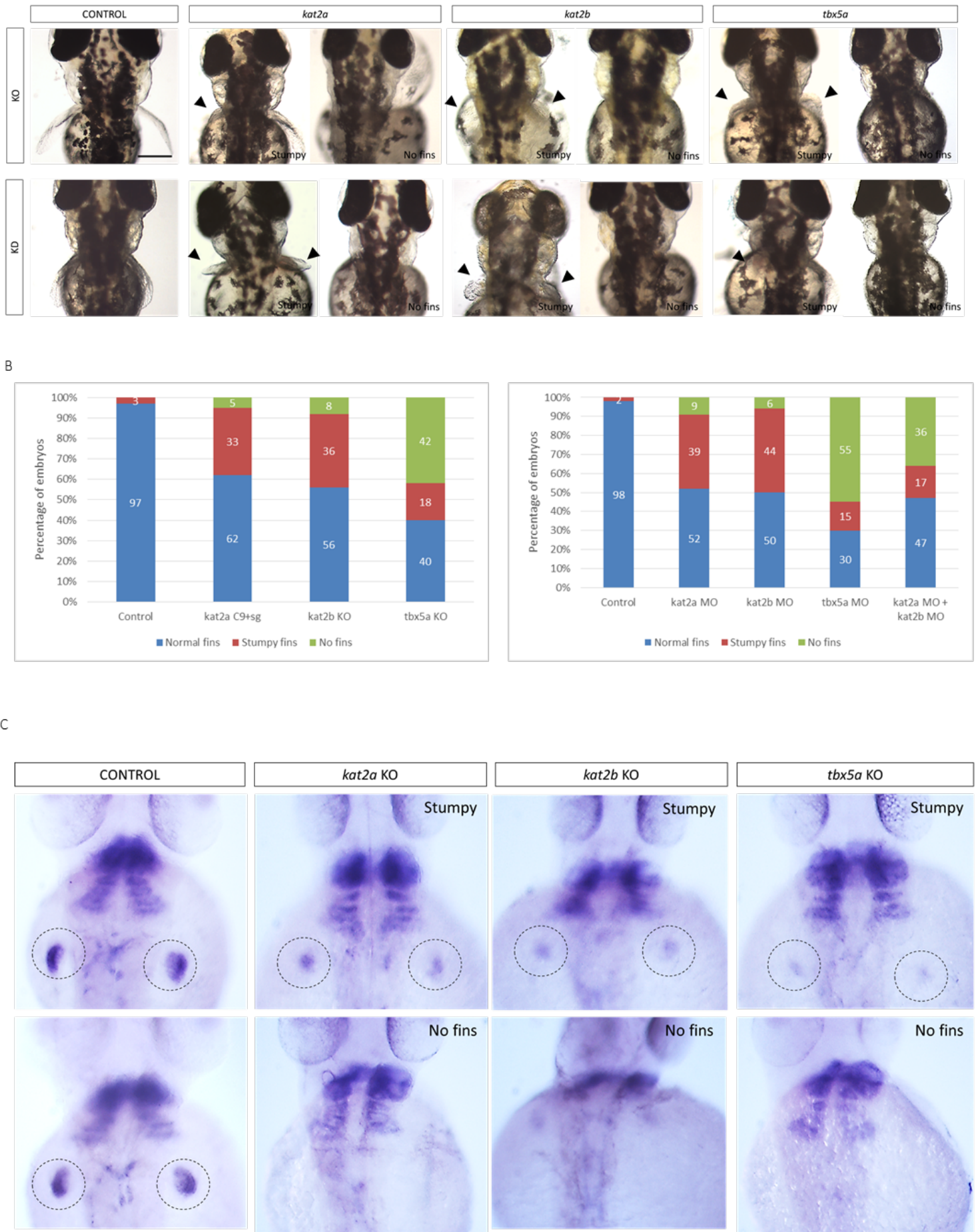

Figure 6. Fin development is affected in kat2a and kat $2 b \mathrm{KO} / \mathrm{KD}$ embryos. (A) Representative images of 72 hpf control embryos (presenting fully-developed pectoral fins) and kat $2 \mathrm{a}$, kat $2 \mathrm{~b}$ and tbx $5 \mathrm{~b} \mathrm{KO} / \mathrm{KD}$ embryos where fin development is abnormal (examples of embryos with stumpy fin/s or embryos with no fins are shown for each phenotype). Black arrows 
indicate the presence of shortened, stumpy fins. (B) Distribution of fin phenotypes in Cas9+sgRNA-injected embryos and in morpholino-injected embryos ( $\mathrm{n}=200$ embryos per injection batch). (C) In situ hybridization in $48 \mathrm{hpf}$ embryos using a bmp 4 probe. In embryos presenting stumpy fins, fins are smaller and bmp4 expression level is reduced when compared to controls (bmp4 signal in the fins is highlighted by a dashed circle). No signal is detected in embryos lacking both pectoral fins. Scale $\mathrm{bar}=200 \mu \mathrm{m}$

A
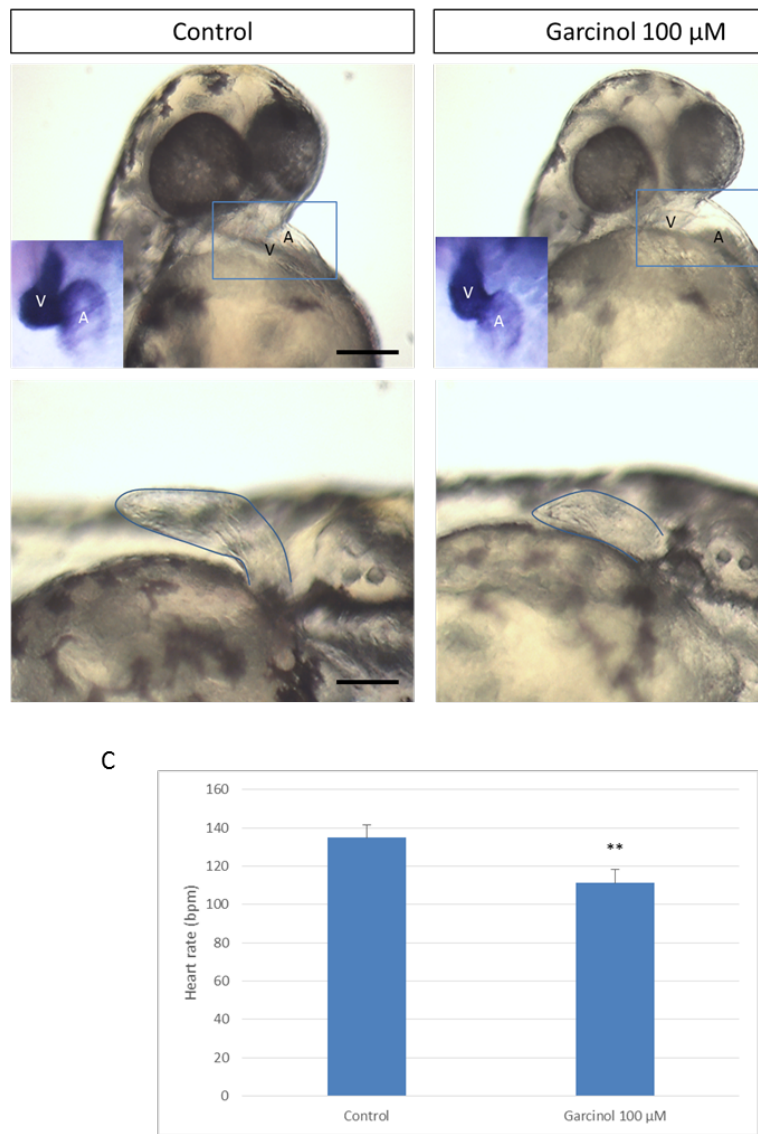

B
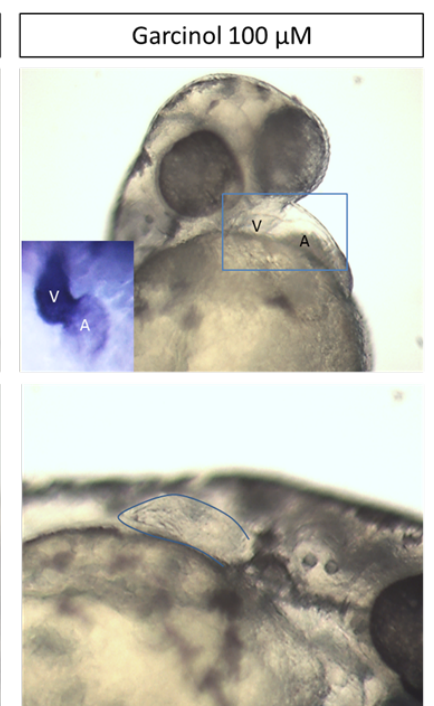

Garcinol $100 \mu \mathrm{M}$

D
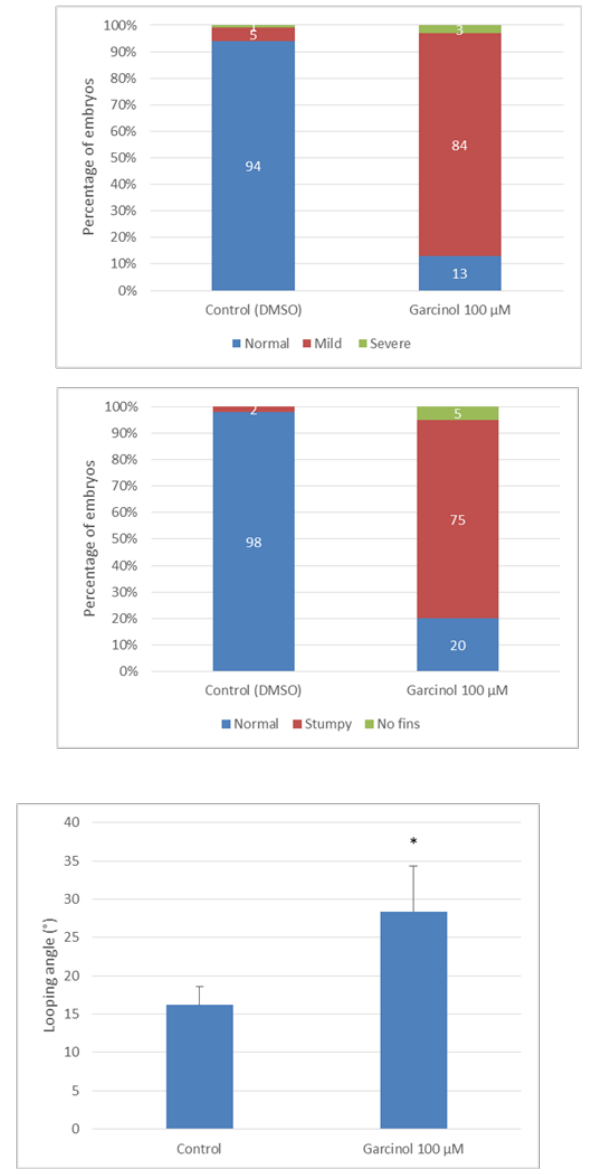

Figure 7. Treatment of zebrafish embryos with acetyl-transferases inhibitor Garcinol also produces heart and fin defects. (A) Images of $48 \mathrm{hpf}$ control embryos treated with Garcinol $100 \mathrm{uM}$. On the upper row, the heart is highlighted using a blue square, and representative in-situ hybridization images using a $\mathrm{cmlc} 2$ probe are shown for each phenotype (bottom left corner, $\mathrm{V}=$ ventricle, $\mathrm{A}=$ atrium). Pectoral fins are shown in the bottom row. (B) Distribution of cardiac and fin phenotypes based on severity in treated embryos ( $\mathrm{n}=200$ embryos per group). (C) Heart beating rate was measured in 48 hpf embryos, indicating the presence of bradycardia in treated embryos. (D) Average looping angle in $48 \mathrm{hpf}$ control and treated embryos. For (C) and (D), data + SEM is represented ( $n=10-20$ embryos per phenotype). Relative to controls, treated embryos were statistically significant $(* * \mathrm{P}<0.001$, or $* \mathrm{P}<0.01)$. Scale bar $=200 \mu \mathrm{m}$

\section{Tbx5a K339Q acetyl-mimic is able to partially rescue the kat2a/kat2b KO phenotype}

Our biochemical and genetic studies suggest that $k a t 2 a$ and $k a t 2 b$ regulate $t b x 5 a$ during heart and forelimbs development. To further confirm the in vivo link between these factors, we performed a rescue experiment of the kat $2 a \mathrm{KO}$ phenotype using a $t b x 5 a$ acetyl-mimic transcript (tbx5a K339Q). As shown in Fig. 8, co-injection of Cas9+kat2a sgRNA along with the acetyl-mimic mRNA leads to an increase in the number of embryos showing a normal cardiac phenotype in comparison with their $\mathrm{KO}$ counterparts $(72 \%$ in the rescue group vs $52 \%$ in the knockout group). When analysing the fin phenotype in the rescue and knockout groups, we also found a similar increase in the percentage of embryos showing normal fins in the rescue group when compared with the knockout group (75\% vs 59\% respectively). 
Overall, these data support the fact that $t b x 5 a$ in vivo acetylation is required for proper cardiac and limb development in zebrafish.

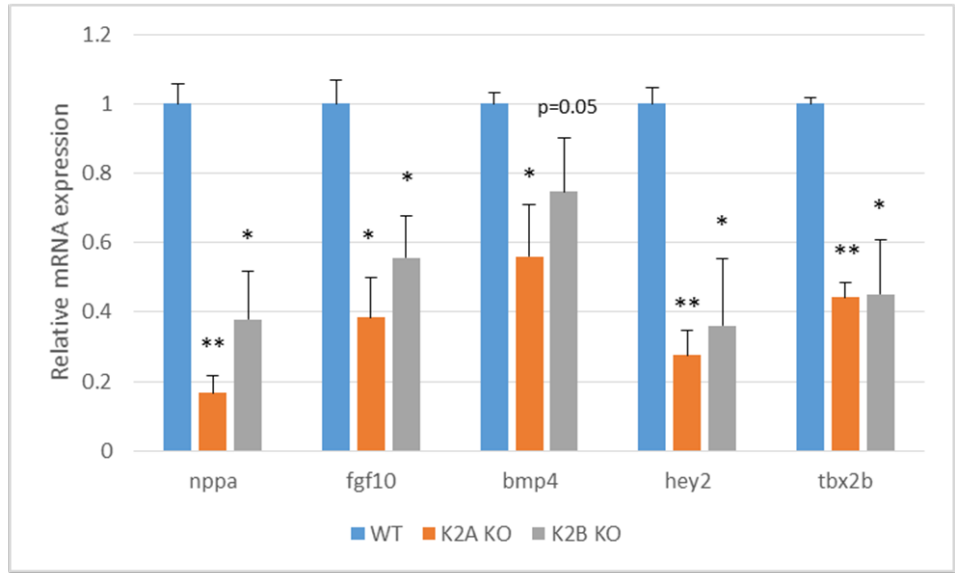

B

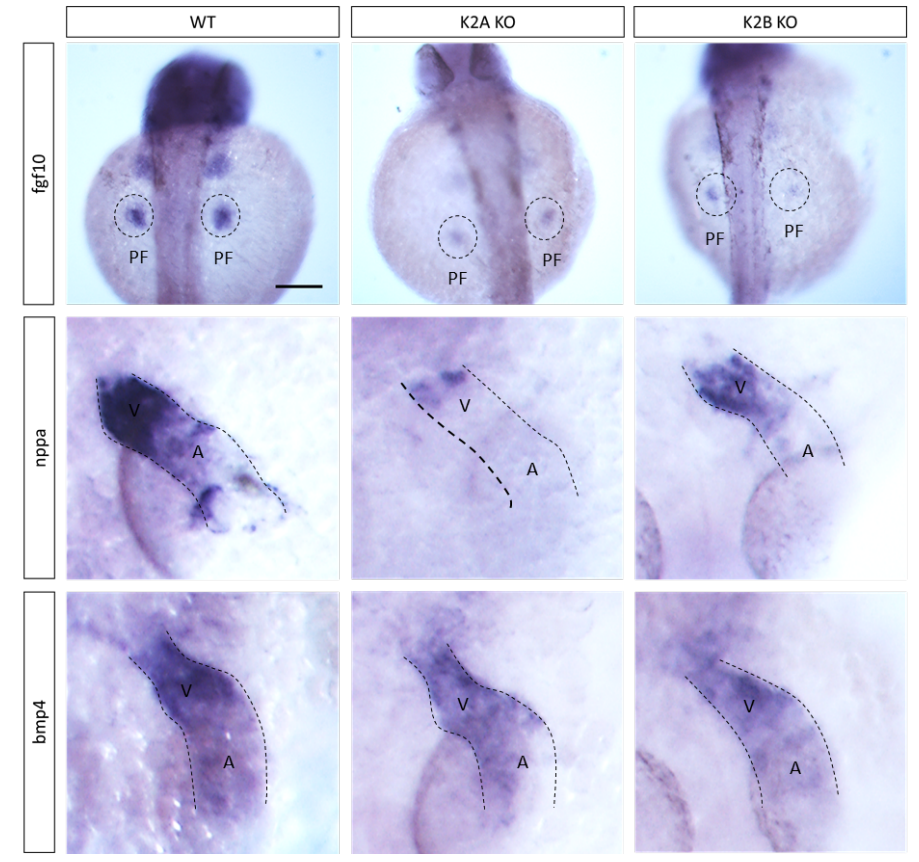

Figure 8. Effect of $k a t 2 a / k a t 2 b \mathrm{KO}$ in $t b x 5 a$ target genes during zebrafish development. (A) qPCR analysis showing downregulation of nppa, fgflo, bmp 4, hey 2 and tbx $2 b$ at $32 \mathrm{hpf}$ in kat $2 a$ and $k a t 2 b \mathrm{KO}$ embryos. Mean + SEM is represented $(\mathrm{n}=3)$. ${ }^{*} \mathrm{P}<0.005$, or $* \mathrm{P}<0.05$. (B) In-situ hybridization using fgfl0, nppa and bmp4 probes was performed on $32 \mathrm{hpf}$ $k a t 2 a$ or kat $2 b \mathrm{KO}$ embryos. Pectoral fins $(\mathrm{PF})$ are highlighted by a dashed circle, and the heart is highlighted by dashed line ( $\mathrm{V}=$ ventricle, $\mathrm{A}=$ atrium).

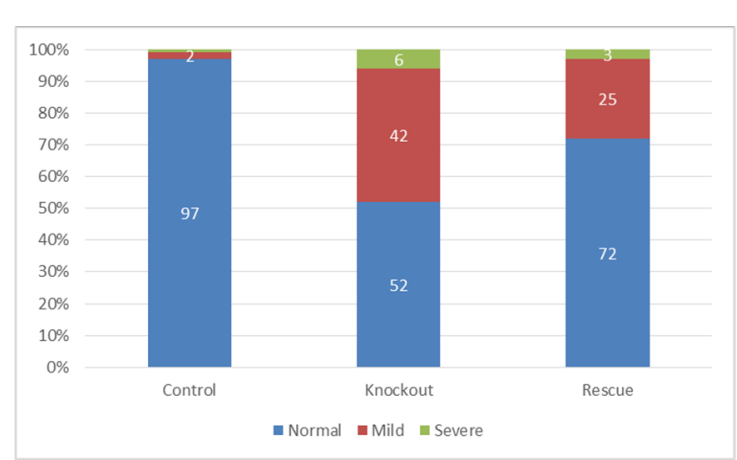

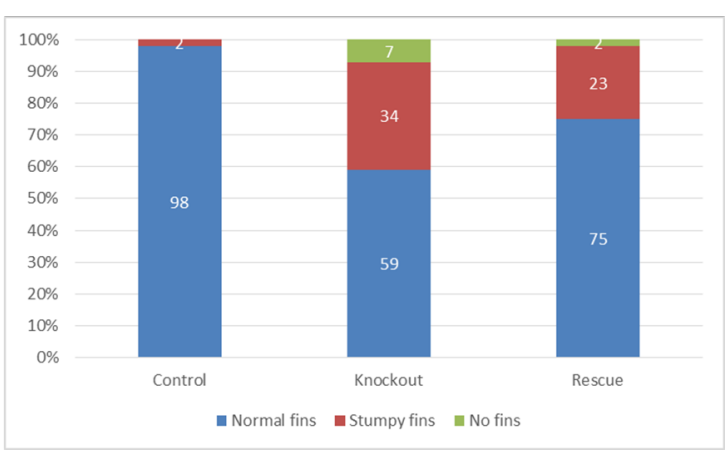


Figure 9. Rescue of the kat2a KO phenotype was performed by injecting in vitro synthesized tbx5a K339Q mRNA. (A) Distribution of cardiac phenotypes (48 hpf) based on severity in control, knockout and rescue embryos is shown. (B) Distribution of fin phenotypes ( $72 \mathrm{hpf}$ ) based on severity ( $\mathrm{n}=200$ embryos per injection batch). $\mathrm{p}<0.01$, chi-square test.

\section{DISCUSSION}

The function of TBX5 is primarily modulated by its interacting proteins, but as recent studies suggest it can also be modulated by posttranslational modifications such as acetylation (Lewandowski et al. 2014) and sumoylation (Beketaev et al. 2014). We have identified acetyl modification of TBX5 and showed that modification is linked to heart and limb development. In cell transfection assays, we showed that both KAT2A and KAT2B physically interact with and acetylate TBX5, and that acetylation enhanced its transcriptional potential. Recent studies have linked p300 with the acetylation of Tbx5 (Lewandowski et al. 2014). However, we failed to detect p300-mediated acetylation of TBX5 in our experiments. The apparent disparity could be due to the different cell lines used in the assay systems or a very low level of acetylation by p300 that we were unable to detect. Acetylation and deacetylation regulate Tbx5-mediated gene transcription during early cardiogenesis (Lewandowski et al. 2014). Previous studies suggested TIP60, a MYST family histone acetyltransferase, associates with Tbx5 to activate the SRF promoter and that requires a functional histone acetyltransferase domain (Barron et al. 2005). Acetyl modification of other cardiac transcription factors such as GATA4 (Miska et al. 2001) and MEF2C (Kuninger et al. 2007) is thought to play an important role in cardiomyocyte differentiation.

To map the acetyl lysine residue in TBX5, we employed on-line prediction tools such as PredMod, PAIL and PHOSIDA. Whilst the prediction varies considerably, Lys234 was picked up by all three tools. However, our subsequent mutagenesis studies ruled out Lys 234 as a key target of acetylation. Similar attempts by Lewandowski et al to map the key lysine residues in TBX5 were unsuccessful (Lewandowski et al. 2014). Acetylation of lysine residues in non-histone proteins is mostly carried out by acetyltransferases p300 and KAT2B. Thus far a specific molecular signature for acetylation has not been determined. Based on P53 acetylation, a consensus sequence K/RXKK was suggested for acetyltransferase p300 (Gu and Roeder 1997). The HAT domain of Tetrahymena GCN5, a PCAF homologue, binds a specific site containing a sequence G-K-X-P on histone proteins (Rojas et al. 1999). However, these motifs are not present in most of the acetylated non-histone proteins including TBX5. Using sequence alignment around the key lysine residues of KAT2B/2Amediated acetylated proteins, we derived a lysine rich consensus motif $K-X-X-K-K / R-P / L / K$ as putative acetylation sites. TBX5 does not show a full-length motif but it does contain two partial motifs K-K/R-P/L/K at positions 325 and 339 respectively. These two partial motifs also overlap with recognition sequences obtained for p300 and GCN5 (Gu and Roeder 1997, Rojas et al. 1999). Site directed mutagenesis studies of the lysine residues in these motifs indicated that acetylation occurs on Lys339, which is highly conserved across species.

TBX5 has two nuclear localization signals: NLS1 (aa78-90) and NLS2 (aa325-340), which act in a cooperative fashion (Collavoli et al. 2003). Lys 339 is located in NLS2 of TBX5. Our results demonstrate that the acetylation-deficient mutant TBX5-K339R accumulates 
mostly in the cytoplasm, and treatment with TBX5-WT with Garcinol led to the same defect in cellular distribution. This impairment in nuclear localization could be due to two different reasons: a defect in nuclear import or an enhanced nuclear export. Leptomycin B treatment of the acetyl-deficient TBX5-K339R variant lead to its nuclear accumulation, thus suggesting this mutant is still able to get into the nucleus, but it is not retained inside. The nuclear export of TBX5 occurs through exportin CRM1, which interacts with an evolutionarily conserved nuclear export signal (NES) at 152-160 (Kulisz and Simon 2008). Indeed, co-localization studies showed that TBX5-K339R and CRM1 interact outside the nucleus. In order to understand how acetylation could regulate CRM1-mediated export of TBX5, we performed pull-down studies. The results suggest that the acetyl-deficient TBX5K339R presents higher affinity for CRM1 than its WT counterpart.

The TBX5 acetylation site within NLS2, when not acetylated, appears to promote TBX5 nuclear export via the CRM1-pathway. This kind of mechanism has been described elsewhere: acetylation of several proteins such as HNF-4, POP-1 or CtBP2 within their respective NLS leads to their nuclear retention by inhibiting CRM1-mediated export (Soutoglou et al. 2000, Gay et al. 2003, Zhao et al. 2006). As acetylation enhances its nuclear retention, that increases the nuclear pool of TBX5 leading to enhanced transcriptional activity. Lack of TBX5 acetylation increases its affinity for the CRM1 exportin, which leads to its efficient nuclear export and subsequently reduces its transcriptional activity.

To examine the in vivo role of kat $2 a$ and kat $2 b$ we performed gene knock-down and knockout experiments in zebrafish at early stages of development and examined the phenotypic consequences. Both morpholino-mediated knockdown and CRISPR-Cas-mediated knockout of $k a t 2 a$ or $k a t 2 b$ produced unlooped elongated hearts and stumpy fin phenotypes, similar to those observed in $t b x 5$ morphants and mutants, suggesting that both may work in a common pathway of heart and limb development. The main difference between the phenotype of $k a t 2 a / k a t 2 b$ mutant embryos and that of $t b x 5 a$ mutants lies in the severity of the phenotype. Most of kat $2 a$ and kat $2 b$ single knockouts present a mild cardiac phenotype, whereas most of tbx $5 a$ mutants show a more severe cardiac phenotype. Several publications have previously suggested that Kat proteins present functionally overlapping roles (Jin et al. 2011, Jin et al. 2014), and mice lacking both Kat2a and Kat2b die at an earlier stage than Kat2a single KO mice (Yamauchi et al. 2000). Based on our data, knockdown of both zebrafish kat proteins also lead to the presence of a greater number of embryos presenting a severe phenotype, supporting a potential redundant effect between kat $2 a$ and $k a t 2 b$.

$k a t 2 a$ and $k a t 2 b \mathrm{KO} / \mathrm{KD}$ also present abnormal fin development, one of the main phenotypic features of $t b x 5 a$ mutants. Most of kat $2 a$ or kat $2 b$ mutants developed fins but they were abnormal in shape or size, whereas $t b x 5 a$ mutants lacked both fins in most cases. Knockdown of both kat proteins increased the number of embryos lacking both fins, a result in concordance with the kat $2 a$ and kat $2 b$ redundancy. In addition to this, we have also further substantiated the role of acetylation during development by inhibiting the acetyltransferase activity on zebrafish embryos. Treatment with Garcinol (HAT inhibitor) lead to cardiac and fin defects similar to those found on kat $2 a$ and kat $2 b$ knock-down and knock-out embryos. 
Most of the embryos treated with Garcinol showed mild defects, suggesting this that this compound do not completely abolish the acetyltransferase activity (Garcinol is predicted to exclusively target KAT2B and p300 acetyltransferases).

In order to provide evidence that loss of $t b x 5 a$ acetylation is indeed responsible for the $k a t 2 a / k a t 2 b$ phenotype, we performed qPCR and ISH analysis of typical $t b x 5 a$ target genes, including nppa, fgf10, bmp 4, hey 2 and $t b \times 2 b$, which have been showed to be downregulated in a $t b x 5 a \mathrm{KO}$ context in the past (Parrie et al. 2013, Puskaric et al. 2010, Zhao et al. 2015). Both approaches confirmed the down-regulation of $t b x 5 a$ targets in both kat $2 a$ and kat $2 b \mathrm{KO}$ embryos. To further support an in vivo link between $t b x 5 a$ and kat 2 proteins, we attempted to rescue the kat $2 a \mathrm{KO}$ phenotype using a $t b x 5 a \mathrm{~K} 339 \mathrm{Q}$ acetyl-mimic. Glutamine is an effective mimic of acetylated lysine due to the similarity of charge and chemical structure, and it can function as acetylated lysine in vivo (Li et al. 2002, Wang and Hayes 2008). Indeed, injection of K339Q variant lead to a partial rescue of both the cardiac and fin phenotype in kat2a KO embryos. These results suggest that loss of $t b x 5 a$ acetylation is mainly responsible for the cardiac and fin abnormalities present in our kat2a/kat $2 b$ knock-down and knock-outs.

In summary, both in vitro and in vivo data support the notion that KAT2A and KAT2B acetyltransferases are tightly linked to TBX5 and control its transcriptional activity, thus leading to abnormal cardiac and upper limb development. Our studies suggest that K339 is important and provide insight into a new mechanism of TBX5 activity regulation in which acetylation is required for its nuclear retention and for maintaining a critical concentration inside the nucleus. Although a large number of TBX5 mutations have been identified in cases of HOS, no mutation has been reported at lysine 339 thus far. However, we would predict that non-acetylation of TBX5 would likely cause or contribute to functional deficiency that may lead to HOS in humans.

\section{MATERIALS AND METHODS}

\section{Plasmids Construction}

A FLAG tagged TBX5 PCR product was generated from plasmid pcDNA-TBX5 described previously (Ghosh et al. 2001) and cloned into NheI and BamHI of pcDNA3.1 (Invitrogen) to give rise to pcDNA-TBX5-FLAG. KAT2A-HA and CRM1-HA PCR products were generated from IMAGE clones 6137532 and 5267242 (Source Bioscience, UK) respectively. The PCR products were then digested and cloned into NheI and XhoI sites of pcDNA3.1 to generate pcDNA-KAT2A-HA and pcDNA-CRM1-HA respectively. We generated pcDNAKAT2B-HA by subcloning KAT2B-HA from pBSSK-KAT2B-HA into XhoI and XbaI sites of pcDNA3.1. Generation of promoter-reporter plasmid pGL3-MYH6-I and pGL3-ANF was reported previously (Ghosh et al. 2001, Ching et al. 2005). Plasmid pGL3-CX40 was a gift from Mauro W. Costa. Plasmid pEGFP-TBX5 was generated by cloning TBX5 into Nhe I site of pEGFP-C1 vector (Clontech).

\section{Acetylation}


The wild type or mutant FLAG-tagged TBX5 plasmids were cotransfected with or without pcDNA-p300-FLAG, pcDNA-KAT2B-HA or pcDNA-KAT2A-HA constructs in Cos7 cells. Cell lysates were generated from $48 \mathrm{~h}$ post transfected cells in cell lysis buffer $(150 \mathrm{mM} \mathrm{NaCl}$, $20 \mathrm{mM}$ Tris (pH-7.6), 1\% NP-40, $1 \mathrm{mM}$ PMSF, $1 \mu \mathrm{g} / \mathrm{mL}$ aprotinin, $1 \mu \mathrm{g} / \mathrm{mL}$ pepstatin, 1 $\mu \mathrm{g} / \mathrm{mL}$ leupeptin, $0.5 \mathrm{mM}$ EDTA and $0.25 \%$ deoxycholate). TSA $(0.3 \mu \mathrm{M})$ was added to the plates $2 \mathrm{~h}$ prior to harvest. Initially, cells were harvested from two $100 \mathrm{~mm}$ plates in PBS, pelleted down and resuspended in 200ul of lysis buffer. The cell suspensions were then placed under ice for $1 \mathrm{~h}$ followed by brief sonication. TBX5-FLAG proteins were partially purified on Anti-FLAG M2 affinity gels (Sigma), extracted with NuPAGE LDS sample buffer (Life Technologies) and fractionated on NuPAGE 10\% Bis-Tris gels (Life technologies) followed by Western blotting. Acetylated proteins were detected using antiacetylated lysine antibody (Cell Signaling). FLAG-TBX5 proteins were detected on the same blot using Anti-FLAG M2 antibody (Sigma). The expression level of KAT2B/KAT2A in the extracts was detected using monoclonal Anti-HA antibody (Sigma).

In order to perform in vitro acetylation assays we overexpressed TBX5 as MBP-TBX5 in bacteria and partially purified on Amylose resin. We obtained purified recombinant KAT2B protein from Abcam. Acetylation assays were performed as described previously (Kuninger et al. 2007).

\section{Pull-down Assay}

Cell extracts were incubated with 20-30ul of washed Anti-FLAG M2-Affinity gel (Sigma) at $4^{0} \mathrm{C}$. For interaction studies, cell lysates were added to the affinity gel and incubated for $2 \mathrm{~h}$ or overnight in a shaker at $4^{0} \mathrm{C}$. To remove non-specific proteins, beads were washed 6 times in wash buffer (20mM Tris (pH7.6), 150mM NaCl, 0.5\% NP40 and 0.5mM EDTA). Bound proteins were specifically eluted from the beads with wash buffer $(15.0 \mu \mathrm{l})$ containing $3 \mathrm{X}$ FLAG peptide (Sigma). Eluted proteins were fractionated on $10 \%$ Bis-Tris gel followed by Western blotting. FLAG-tagged and HA-tagged proteins were detected using Anti-FLAG M2 antibody (Sigma) and monoclonal Anti-HA antibody (Sigma) respectively. Endogenous levels of Kat2b and Kat2a were detected using anti-KAT2B (Cell Signaling) and antiKAT2A (abcam) antibodies respectively. Anti- $\alpha$ Tubulin (Santa Cruz) was used to detect housekeeping protein tubulin. Detection of CRM1 protein was carried out with anti-CRM1 antibody (Santa Cruz). Semi-quantitative analysis of the bands was performed using the software ImageJ.

\section{Cell Culture, Transfection and Reporter Assay}

Cos7 and H9c2 cells were transfected using Polyfect (Qiagen) according to the manufacturer's protocol. C2C12 cells were nucleofected using nucleofector kit V (Lonza). For pull-down assays we used Cos7 cells and transfections were performed using $6 \mu \mathrm{g}$ of each plasmid DNA in $100 \mathrm{~mm}$ tissue culture dishes. Reporter assays were carried out in H9c2 cells using $1.5 \mu \mathrm{g}$ reporter plasmid pGL3-MYH6-I, $1.0 \mu \mathrm{g}$ of pcDNA-TBX5 or pcDNA-TBX5K339R, $1.0 \mu \mathrm{g}$ pcDNA-KAT2B or pcDNA-KAT2A, and 4 ng of pRL-TK was used as an internal control to normalize variation in transfection efficiency between the 
plates. Luciferase assays were carried out on cell lysates using dual luciferase assay kit (Promega) in duplicates and each experiment was carried out at least three times. Values were obtained as means \pm S.D. Significance analysis was performed using student's $t$ test.

\section{Site-Directed Mutagenesis}

Site directed mutagenesis was carried out using the QuickChange Site Directed Mutagenesis Kit (Agilent Technologies). pcDNA-TBX5-FLAG was used as a template to generate the mutants K234R, K325R, K327R, K339R and K340R.

\section{RT-PCR Analysis}

Wild type zebrafish embryos were isolated and collected at various developmental stages; they were subsequently dissected using surgical microlances to produce tail and head/thorax sections. The dissected sections were collected on dry ice and immediately frozen for storage prior to processing. Total RNA was isolated from pools of 50-100 zebrafish embryos of the respective section using TRI-Reagent according to manufacturer's instructions. DNase treatment was carried out at $37^{\circ} \mathrm{C}$ for 30 minutes. First strand cDNAs were synthesised from $1 \mu \mathrm{g}$ of Total RNA with M-MuLV Reverse Transcriptase in a final volume of $20 \mu 1$ including $1 \mathrm{x}$ RT Buffer, $1 \mathrm{Mm}$ dNTPs, RNase Inhibitor, $2 \mu \mathrm{l}$ of random hexamers and made up with DEPC water. The reaction mixture was incubated at $42^{\circ} \mathrm{C}$ for 1 hour and the enzyme deactivated at $90^{\circ} \mathrm{C}$ for 10 minutes. The following primers were used for zebrafish to detect the expression of kat $2 a$ and $k a t 2 b$ and a reference control gene $\beta$-actinl.

kat2bF: GGTACGAGGCCACACAGATT ; kat2bR: GTAGGAAACATCCGGAAGCA

kat2aF: CCTAAGCCAACAGCAATGGT ; kat2aR: GCTTGGTGTCCGTGTCTTCT

$\beta$-actin1F: ACATCAGCATGGCTTCTGCT; $\beta$-actin1R: GTGTACAGAGACACCCTGGC.

PCR amplification was performed in $25 \mu$ reactions containing final concentration of $1 \mathrm{x}$ standard Taq reaction buffer, $200 \mu \mathrm{M}$ dNTPs, $0.2 \mu \mathrm{M}$ primers, $1.25 \mathrm{U}$ of Taq enzyme, $1 \mu \mathrm{l}$ of resultant cDNA and made up with Nuclease- free water. Thermocycling conditions were an initial denaturation of 30 seconds at $95^{\circ} \mathrm{C}$ followed by 35 cycles of 30 seconds of denaturation, 30 seconds annealing at $59^{\circ} \mathrm{C}$ and 50 seconds of elongation at $68^{\circ} \mathrm{C}$ followed by a final extension time of 5 minutes at $68^{\circ} \mathrm{C}$. RT-PCR products were resolved by electrophoresis on $1.5 \%$ agarose gels and visualized with ethidium bromide.

\section{Immunofluorescence and cell imaging:}

Cos7 cells were grown on coverslips in 6 well plates overnight and transfected with TBX5WT-FLAG or TBX5-K339R-FLAG constructs using polyfect (Qiagen). C2C12 cells were transfectd using Amaxa Cell Line Nucleofector kit $\mathrm{V}$ according to manufacturer's protocol (Lonza). When required, transfected cells were treated with $10 \mathrm{nM}$ of leptomycin B or $20 \mu \mathrm{M}$ of Garcinol 16 hours before fixation. Twenty four hours posttransfection cells were washed in 
phosphate buffer saline (PBS, $\mathrm{pH} 7.4$ ), fixed with 4\% paraformaldehyde for $10 \mathrm{mins}$ and washed in PBS three times, 5 minutes each. Cells were then permeabilized with $0.25 \%$ Triton $\mathrm{X}-100$ in PBS for 5 minutes washed in PBS three times and incubated in 10\% BSA in PBS for 1 hour at room temperature to supress non-specific binding of IgG. After blocking the cells were washed in PBS and incubated with mouse monoclonal antibody against FLAG (Sigma, F1804, dilution 1:300) or with rabbit anti-human CRM1 antibody (SantaCruz, sc74454, dilution 1:50) in PBS containing 3\% BSA for 2 hours at room temperature. Cells were washed three times in PBS each for 5 minutes and incubated in 3\% BSA in PBS containing secondary antibody Alexa Fluor 488 goat anti-rabbit IgG (Life Technology, $2 \mu \mathrm{g} / \mathrm{ml}$ ) for 45 minutes at room temperature. Then cells were washed three times in PBS each for 5 minutes and the washed coverslips were mounted on glass slides containing a small drop of mounting media containing DAPI (Vectashield). Laser scanning confocal microscopy was performed on LSM 880F (Carl Zeiss, Oberkochen, Germany) using a 63X 1.4 oil objective.

\section{Knockdown and Knockout Experiment in Zebrafish}

Wildtype zebrafish were raised and maintained as described by Westfield (Westfield 1993). Initially to assess the function/phenotype of kat $2 a$ and kat $2 b$ we used antisense morpholino oligonucleotides to block translation. These were designed and made by Gene Tools: kat $2 a$ 5'-TCCGCCATAACAGCAGCCACACTTC-3' GATCCCCGTGCTTTCCGACATCGAA-3'. Two additional morpholinos were designed to target the intron1-exon2 splice sites in both genes respectively: kat2a splice 5'ATTCGCCTGAGATAAGAAGAGTGCA-3', kat2b splice 5'TCAGCCTGTGTGAAAGAAAGAAGAGTGCA-3' (Gene Tools). All morpholinos were injected at 1-8 cell stage and mismatch morpholino-injected siblings were used as controls. In order to generate kat2a, kat $2 b$ and $t b x 5 a$ knockouts using the CRISPR-Cas system, sgRNAs for each target gene were designed using the web tool CHOPCHOP (http://chopchop.cbu.uib.no/). DNA template for sgRNA transcription was generated using a cloning-free method previously described (Moreno-Mateos et al. 2015). Approximately 150 ng of template was used for sgRNA in vitro transcription using a MAXIscript T7 transcription kit (Ambion) and quality was examined using a 2100 Bioanalyzer (Agilent Technologies). Subsequently, $100 \mathrm{pg}$ of sgRNA and $500 \mathrm{pg}$ of Cas9 protein (NEB) were injected into 1-cell stage zebrafish embryos and Cas9-only injected embryos were used as controls. The injected embryos and controls were kept at $28.5^{\circ} \mathrm{C}$ for 2 or 3 days postfertilization. The images of live 2/3-day-postfertilization zebrafish were captured using a Zeiss Stereo Lumar V12 microscope with a camera. Phenotypic severity was categorized into three groups: normal, mild and severe.

\section{Drug treatment}

In order to chemically inhibit the acetyltransferase activity, we used Garcinol (Santa-Cruz Biotechnology). First, pools of 10-15 embryos were treated using a range of different concentrations $(0.1$ to $500 \mu \mathrm{M})$ to find which gave a reproducible and consistent phenotype without compromising viability. Treatments were carried out for 24 hours starting at 20-24 
hpf (initial stages of cardiogenesis). Embryos were washed at $48 \mathrm{hpf}$ to remove the drug and they were subsequently screened for cardiac or fin defects.

\section{Quantitative PCR}

Expression levels of $t b x 5 a$ target genes were measured using a 7500 Real-Time PCR system (Applied Biosystems). Total RNA was extracted from 32 hpf kat $2 a$, kat $2 b$ and $t b x 5 a \mathrm{KO}$ embryos for posterior cDNA synthesis and qPCR analysis. Two well established zebrafish housekeeping genes, efl $a$ and $l s m 12 b$, were used for normalization. The following primers were used for the detection of nppa, fgflo, bmp 4, hey $2, t b x 2 b$, efla and $l s m 12 b$ expression levels:

nppaF: CAGACACAGCTCTGACAGCAACAT; nppaR:

CTCTGTGTGTCAAATCCATCCGAG

fgf10F: CTCATCGTCTGCCGTGGTG; fgf10R: GTCAATGCCGAAATCCCTC

bmp4F: CACAGTATCTGCTCGACCTCTA; bmp4R:

GATATGAGTTCGTCCTCTGGGATG

hey2F: GAAAGAAGCGGAGAGGGATCATTG; hey2R:

AGAAGTCCATGGCCAGAGAATGAG

tbx2bF: GTCCCTTTCCCTTTCATCTGTCTC; tbx2bR:

CTGGGAGCTGATAAGGGTTGAATC

eflaF: CGGTGACAACATGCTGGAGG; eflaR: ACCAGTCTCCACACGACCA

1sm12bF; AGTTGTCCCAAGCCTATGCAATCAG; 1sm12bR:

CCACTCAGGAGGATAAAGACGAGTC

\section{Rescue experiment}

For rescue experiments, we synthesised capped mRNA transcripts from the SP6 promoter of linearized plasmid pCS2-TBX5A-K339Q (previously generated by cloning) using mMessage mMachine kit (Ambion). $300 \mathrm{pg}$ of $t b x 5 a$ K339Q mRNA were co-injected with $100 \mathrm{pg}$ of sgRNA and $500 \mathrm{pg}$ of Cas9 protein (NEB) into 1-cell stage zebrafish embryos. Injected embryos were screened 48 and $72 \mathrm{hpf}$ for the presence of cardiac and fin defects.

\section{In-situ hybridization and cardiac looping measurement}

In order to study heart and fin morphology, injected embryos were fixed overnight in $4 \%$ paraformaldehyde solution in PBS (PFA) and subjected to in-situ hybridization according to a method previously described (Thisse and Thisse 2008). Cardiac looping angle was determined by measuring the angle formed by the plane of the atrioventricular junction (AVJ) relative to the anterior/posterior axis of the embryo (Chernyavskaya et al. 2012).

\section{Acknowledgements}


Plasmids pBSSK-KAT2B-HA and pcDNA- p300-FLAG were generous gifts from Dr Peter Rotwein (Oregon, USA).

Conflict of Interest statement: None declared.

\section{Author contributions}

T.K.G., J.J. A-S., S.B and J.D.B designed the research. T.K.G, J.J.A-S, and S.B performed the experiments and analysed the data. T.K.G., J.J.A-S, S.B., and J.D.B wrote the manuscript. A.K., S.L., C.S.R., and T.M were involved in reagent preparation.

\section{Funding}

This work was supported by British Heart Foundation.

\section{References}

Backs, J. and Olson, E. N. (2006) 'Control of cardiac growth by histone acetylation/deacetylation', Circ Res, 98(1), 15-24.

Barron, M. R., Belaguli, N. S., Zhang, S. X., Trinh, M., Iyer, D., Merlo, X., Lough, J. W., Parmacek, M. S., Bruneau, B. G. and Schwartz, R. J. (2005) 'Serum response factor, an enriched cardiac mesoderm obligatory factor, is a downstream gene target for Tbx genes', J Biol Chem, 280(12), 11816-28.

Basson, C. T., Bachinsky, D. R., Lin, R. C., Levi, T., Elkins, J. A., Soults, J., Grayzel, D., Kroumpouzou, E., Traill, T. A., Leblanc-Straceski, J., Renault, B., Kucherlapati, R., Seidman, J. G. and Seidman, C. E. (1997) 'Mutations in human TBX5 [corrected] cause limb and cardiac malformation in Holt-Oram syndrome', Nat Genet, 15(1), 305.

Beketaev, I., Kim, E. Y., Zhang, Y., Yu, W., Qian, L. and Wang, J. (2014) 'Potentiation of Tbx5-mediated transactivation by SUMO conjugation and protein inhibitor of activated STAT 1 (PIAS1)', Int J Biochem Cell Biol, 50, 82-92.

Borghi, S., Molinari, S., Razzini, G., Parise, F., Battini, R. and Ferrari, S. (2001) 'The nuclear localization domain of the MEF2 family of transcription factors shows memberspecific features and mediates the nuclear import of histone deacetylase 4', J Cell Sci, 114(Pt 24), 4477-83.

Chernyavskaya, Y., Ebert, A. M., Milligan, E. and Garrity, D. M. (2012) 'Voltage-gated calcium channel CACNB2 (beta2.1) protein is required in the heart for control of cell proliferation and heart tube integrity', Dev Dyn, 241(4), 648-62. 
Ching, Y. H., Ghosh, T. K., Cross, S. J., Packham, E. A., Honeyman, L., Loughna, S., Robinson, T. E., Dearlove, A. M., Ribas, G., Bonser, A. J., Thomas, N. R., Scotter, A. J., Caves, L. S., Tyrrell, G. P., Newbury-Ecob, R. A., Munnich, A., Bonnet, D. and Brook, J. D. (2005) 'Mutation in myosin heavy chain 6 causes atrial septal defect', Nat Genet, 37(4), 423-8.

Collavoli, A., Hatcher, C. J., He, J., Okin, D., Deo, R. and Basson, C. T. (2003) 'TBX5 nuclear localization is mediated by dual cooperative intramolecular signals', $J \mathrm{Mol}$ Cell Cardiol, 35(10), 1191-5.

Garg, V., Kathiriya, I. S., Barnes, R., Schluterman, M. K., King, I. N., Butler, C. A., Rothrock, C. R., Eapen, R. S., Hirayama-Yamada, K., Joo, K., Matsuoka, R., Cohen, J. C. and Srivastava, D. (2003) 'GATA4 mutations cause human congenital heart defects and reveal an interaction with TBX5', Nature, 424(6947), 443-7.

Gay, F., Calvo, D., Lo, M. C., Ceron, J., Maduro, M., Lin, R. and Shi, Y. (2003) 'Acetylation regulates subcellular localization of the Wnt signaling nuclear effector POP-1', Genes Dev, 17(6), 717-22.

Ghosh, T. K., Packham, E. A., Bonser, A. J., Robinson, T. E., Cross, S. J. and Brook, J. D. (2001) 'Characterization of the TBX5 binding site and analysis of mutations that cause Holt-Oram syndrome', Hum Mol Genet, 10(18), 1983-94.

Ghosh, T. K., Song, F. F., Packham, E. A., Buxton, S., Robinson, T. E., Ronksley, J., Self, T., Bonser, A. J. and Brook, J. D. (2009) 'Physical interaction between TBX5 and MEF2C is required for early heart development', Mol Cell Biol, 29(8), 2205-18.

$\mathrm{Gu}$, W. and Roeder, R. G. (1997) 'Activation of p53 sequence-specific DNA binding by acetylation of the p53 C-terminal domain', Cell, 90(4), 595-606.

Ieda, M., Fu, J. D., Delgado-Olguin, P., Vedantham, V., Hayashi, Y., Bruneau, B. G. and Srivastava, D. (2010) 'Direct reprogramming of fibroblasts into functional cardiomyocytes by defined factors', Cell, 142(3), 375-86.

Jin, Q., Yu, L. R., Wang, L., Zhang, Z., Kasper, L. H., Lee, J. E., Wang, C., Brindle, P. K., Dent, S. Y. and Ge, K. (2011) 'Distinct roles of GCN5/PCAF-mediated H3K9ac and $\mathrm{CBP} / \mathrm{p} 300-$ mediated $\mathrm{H} 3 \mathrm{~K} 18 / 27 \mathrm{ac}$ in nuclear receptor transactivation', Embo J, 30(2), 249-62.

Jin, Q., Zhuang, L., Lai, B., Wang, C., Li, W., Dolan, B., Lu, Y., Wang, Z., Zhao, K., Peng, W., Dent, S. Y. and Ge, K. (2014) 'Gen5 and PCAF negatively regulate interferon- 
beta production through HAT-independent inhibition of TBK1', EMBO Rep, 15(11), 1192-201.

Kulisz, A. and Simon, H. G. (2008) 'An evolutionarily conserved nuclear export signal facilitates cytoplasmic localization of the Tbx5 transcription factor', Mol Cell Biol, 28(5), 1553-64.

Kuninger, D., Lundblad, J., Semirale, A. and Rotwein, P. (2007) 'A non-isotopic in vitro assay for histone acetylation', J Biotechnol, 131(3), 253-60.

Lewandowski, S. L., Janardhan, H. P., Smee, K. M., Bachman, M., Sun, Z., Lazar, M. A. and Trivedi, C. M. (2014) 'Histone deacetylase 3 modulates Tbx5 activity to regulate early cardiogenesis', Hum Mol Genet.

Li, M., Luo, J., Brooks, C. L. and Gu, W. (2002) 'Acetylation of p53 inhibits its ubiquitination by Mdm2', J Biol Chem, 277(52), 50607-11.

Li, Q. Y., Newbury-Ecob, R. A., Terrett, J. A., Wilson, D. I., Curtis, A. R., Yi, C. H., Gebuhr, T., Bullen, P. J., Robson, S. C., Strachan, T., Bonnet, D., Lyonnet, S., Young, I. D., Raeburn, J. A., Buckler, A. J., Law, D. J. and Brook, J. D. (1997) 'Holt-Oram syndrome is caused by mutations in TBX5, a member of the Brachyury (T) gene family', Nat Genet, 15(1), 21-9.

McKinsey, T. A., Zhang, C. L. and Olson, E. N. (2001) 'Control of muscle development by dueling HATs and HDACs', Curr Opin Genet Dev, 11(5), 497-504.

Miska, E. A., Langley, E., Wolf, D., Karlsson, C., Pines, J. and Kouzarides, T. (2001) 'Differential localization of HDAC4 orchestrates muscle differentiation', Nucleic Acids Res, 29(16), 3439-47.

Moreno-Mateos, M. A., Vejnar, C. E., Beaudoin, J. D., Fernandez, J. P., Mis, E. K., Khokha, M. K. and Giraldez, A. J. (2015) 'CRISPRscan: designing highly efficient sgRNAs for CRISPR-Cas9 targeting in vivo', Nat Methods, 12(10), 982-8.

Parrie, L. E., Renfrew, E. M., Wal, A. V., Mueller, R. L. and Garrity, D. M. (2013) 'Zebrafish tbx 5 paralogs demonstrate independent essential requirements in cardiac and pectoral fin development', Dev Dyn, 242(5), 485-502.

Puri, P. L., Sartorelli, V., Yang, X. J., Hamamori, Y., Ogryzko, V. V., Howard, B. H., Kedes, L., Wang, J. Y., Graessmann, A., Nakatani, Y. and Levrero, M. (1997) 'Differential 
roles of p300 and PCAF acetyltransferases in muscle differentiation', Mol Cell, 1(1), $35-45$.

Puskaric, S., Schmitteckert, S., Mori, A. D., Glaser, A., Schneider, K. U., Bruneau, B. G., Blaschke, R. J., Steinbeisser, H. and Rappold, G. (2010) 'Shox2 mediates Tbx5 activity by regulating Bmp4 in the pacemaker region of the developing heart', Hum Mol Genet, 19(23), 4625-33.

Rojas, J. R., Trievel, R. C., Zhou, J., Mo, Y., Li, X., Berger, S. L., Allis, C. D. and Marmorstein, R. (1999) 'Structure of Tetrahymena GCN5 bound to coenzyme A and a histone H3 peptide', Nature, 401(6748), 93-8.

Soutoglou, E., Katrakili, N. and Talianidis, I. (2000) 'Acetylation regulates transcription factor activity at multiple levels', Mol Cell, 5(4), 745-51.

Thisse, C. and Thisse, B. (2008) 'High-resolution in situ hybridization to whole-mount zebrafish embryos', Nat Protoc, 3(1), 59-69.

Wang, X. and Hayes, J. J. (2008) 'Acetylation mimics within individual core histone tail domains indicate distinct roles in regulating the stability of higher-order chromatin structure', Mol Cell Biol, 28(1), 227-36.

Westfield, M. (1993) 'The zebrafish book: a guide for the laboratory use of zebrafsih(Brachydanio rerioI.', University of Oregon Press, Eugene, OR, 4th ed.

Xu, W., Edmondson, D. G., Evrard, Y. A., Wakamiya, M., Behringer, R. R. and Roth, S. Y. (2000) 'Loss of Gcn512 leads to increased apoptosis and mesodermal defects during mouse development', Nat Genet, 26(2), 229-32.

Yamauchi, T., Yamauchi, J., Kuwata, T., Tamura, T., Yamashita, T., Bae, N., Westphal, H., Ozato, K. and Nakatani, Y. (2000) 'Distinct but overlapping roles of histone acetylase PCAF and of the closely related PCAF-B/GCN5 in mouse embryogenesis', Proc Natl Acad Sci U S A, 97(21), 11303-6.

Yanazume, T., Hasegawa, K., Morimoto, T., Kawamura, T., Wada, H., Matsumori, A., Kawase, Y., Hirai, M. and Kita, T. (2003) 'Cardiac p300 is involved in myocyte growth with decompensated heart failure', Mol Cell Biol, 23(10), 3593-606.

Yang, X. J., Ogryzko, V. V., Nishikawa, J., Howard, B. H. and Nakatani, Y. (1996) 'A p300/CBP-associated factor that competes with the adenoviral oncoprotein E1A', Nature, 382(6589), 319-24. 
Yao, T. P., Oh, S. P., Fuchs, M., Zhou, N. D., Ch'ng, L. E., Newsome, D., Bronson, R. T., Li, E., Livingston, D. M. and Eckner, R. (1998) 'Gene dosage-dependent embryonic development and proliferation defects in mice lacking the transcriptional integrator p300', Cell, 93(3), 361-72.

Zhao, L. J., Subramanian, T., Zhou, Y. and Chinnadurai, G. (2006) 'Acetylation by p300 regulates nuclear localization and function of the transcriptional corepressor CtBP2', $J$ Biol Chem, 281(7), 4183-9.

Zhao, X., Jiang, B., Hu, H., Mao, F., Mi, J., Li, Z., Liu, Q., Shao, C. and Gong, Y. (2015) 'Zebrafish cul4a, but not cul4b, modulates cardiac and forelimb development by upregulating tbx5a expression', Hum Mol Genet, 24(3), 853-64. 